\title{
The Cesàro space of Dirichlet series and its multiplier algebra
}

\author{
J. Bueno-Contreras $^{\text {a }}$, G.P. Curbera ${ }^{\text {b,* }}$, O. Delgado ${ }^{c}$ \\ ${ }^{a}$ Departamento de Análisis Matemático 8 IMUS, Universidad de Sevilla, Calle Tarfia s/n, Sevilla 41012, Spain \\ ${ }^{\mathrm{b}}$ Facultad de Matemáticas \& IMUS, Universidad de Sevilla, Calle Tarfia s/n, Sevilla 41012, Spain \\ ${ }^{\mathrm{c}}$ Departamento de Matemática Aplicada I, E. T. S. de Ingeniería de Edificación, Universidad de Sevilla, Avenida de Reina \\ Mercedes 4 A, Sevilla 41012, Spain
}

Keywords:

Spaces of Dirichlet series

Cesàro sequence space

Multipliers

\begin{abstract}
A B S T R A C T
We consider the space $\mathcal{H}\left(\right.$ ces $\left._{p}\right)$ of all Dirichlet series whose coefficients belong to the Cesàro sequence space $c e s_{p}$, consisting of all complex sequences whose absolute Cesàro means are in $\ell^{p}$, for $1<p<\infty$. It is a Banach space of analytic functions, for which we study the maximal domain of analyticity and the boundedness of point evaluations. We identify the algebra of analytic multipliers on $\mathcal{H}\left(\right.$ ces $\left._{p}\right)$ as the Wiener algebra of Dirichlet series shifted to the vertical half-plane $\mathbb{C}_{1 / q}:=\{s \in \mathbb{C}: \Re s>$ $1 / q\}$, where $1 / p+1 / q=1$.
\end{abstract}

\section{Introduction}

Several spaces of Dirichlet series have been studied in recent years. Hedenmalm, Lindqvist and Seip introduced in [17] the Hilbert space of Dirichlet series $\mathcal{H}$, consisting of all Dirichlet series

$$
f(s):=\sum_{n=1}^{\infty} a_{n} n^{-s}, \quad s \in \mathbb{C},
$$

with square summable coefficients, $\left(a_{n}\right)_{n=1}^{\infty} \in \ell^{2}$. They used it for solving a problem discussed by Beurling on complete sequences in the space $L^{2}(0,1)$. Due to the Cauchy-Schwarz inequality, each $f \in \mathcal{H}$ defines an analytic function on the vertical half-plane $\mathbb{C}_{1 / 2}:=\{s \in \mathbb{C}: \Re(s)>1 / 2\}$. The space $\mathcal{H}$ becomes a Banach space of analytic functions on $\mathbb{C}_{1 / 2}$ when endowed with the norm

\footnotetext{
th The authors acknowledge the support of MTM2015-65888-C4-1-P, MINECO (Spain).

代你 This work is part of the PhD thesis of José Jorge Bueno Contreras, defended in the University of Sevilla under the direction of Guillermo P. Curbera and Olvido Delgado.

* Corresponding author.

E-mail addresses: bueno@cimat.mx (J. Bueno-Contreras), curbera@us.es (G.P. Curbera), olvido@us.es (O. Delgado).
} 


$$
\|f\|_{\mathcal{H}}:=\left\|\left(a_{n}\right)_{n=1}^{\infty}\right\|_{\ell^{2}}, \quad f \in \mathcal{H}
$$

The Hardy spaces of Dirichlet series $\mathcal{H}^{p}$, for $1 \leq p<\infty$, were introduced by Bayart in [6]. They are given by the completion of the space of Dirichlet polynomials $P(s):=\sum_{n=1}^{N} a_{n} n^{-s}$ for the norm

$$
\|P\|_{\mathcal{H}^{p}}:=\left(\lim _{T \rightarrow \infty} \frac{1}{2 T} \int_{-T}^{T}|P(i t)|^{p} d t\right)^{1 / p}
$$

The space $\mathcal{H}$ corresponds to $\mathcal{H}^{p}$ for $p=2$. In [19], McCarthy considered the weighted Hilbert spaces of Dirichlet series

$$
\mathcal{H}_{\alpha}:=\left\{f(s)=\sum_{n=2}^{\infty} a_{n} n^{-s}: \sum_{n=2}^{\infty}\left|a_{n}\right|^{2}(\log n)^{\alpha}<\infty\right\},
$$

for $\alpha<0$, endowed with the norm

$$
\|f\|_{\mathcal{H}_{\alpha}}:=\left\|\left(a_{n}(\log n)^{\alpha / 2}\right)_{n=2}^{\infty}\right\|_{\ell^{2}}, \quad f \in \mathcal{H}_{\alpha} .
$$

More recently, Bailleul and Lefèvre have studied certain classes of Bergman-type spaces of Dirichlet series, $\mathscr{A}_{\mu}^{p}$ and $\mathscr{B}^{p}$, for $1 \leq p<\infty$, [4]. Another type of weighted Hilbert spaces of Dirichlet series $\mathscr{D}_{\alpha}$, for $\alpha>0$, has been considered by Bailleul and Brevig in [3]. It should be highlighted that the spaces $\mathcal{H}, \mathcal{H}^{p}, \mathcal{H}_{\alpha}, \mathscr{A}_{\mu}^{p}$, $\mathscr{B}^{p}, \mathscr{D}_{\alpha}$ are all Banach spaces of analytic functions on the vertical half-plane $\mathbb{C}_{1 / 2}$.

A deep feature of Dirichlet series is their product. The pointwise product $f(s) \cdot g(s)$ of two Dirichlet series $f(s)=\sum_{n=1}^{\infty} a_{n} n^{-s}$ and $g(s)=\sum_{n=1}^{\infty} b_{n} n^{-s}$ is, in the appropriate domain, the Dirichlet series $h(s)=\sum_{n=1}^{\infty} c_{n} n^{-s}$ whose coefficients $c=\left(c_{n}\right)_{n=1}^{\infty}$ are given by the Dirichlet convolution $c:=a \cdot b$ of the sequences $a=\left(a_{n}\right)_{n=1}^{\infty}$ and $b=\left(b_{n}\right)_{n=1}^{\infty}$, that is,

$$
c_{n}=(a \cdot b)_{n}:=\sum_{k \mid n} a_{k} b_{\frac{n}{k}}, \quad n \geq 1,
$$

where $k \mid n$ denotes that $k$ is a divisor of $n$.

Given a space $\mathcal{E}$ of Dirichlet series, a multiplier on $\mathcal{E}$ is an analytic function $f$ with the property that $f g \in \mathcal{E}$ for every $g \in \mathcal{E}$. The multiplier algebra of $\mathcal{E}$ is the set of all multipliers on $\mathcal{E}$; we denote it by $\mathcal{M}(\mathcal{E})$. Note that $\mathcal{M}(\mathcal{E}) \subseteq \mathcal{E}$ whenever $\mathbf{1} \in \mathcal{E}$. Neither of the spaces $\mathcal{H}, \mathcal{H}^{p}, \mathcal{H}_{\alpha}, \mathscr{A}_{\mu}^{p}, \mathscr{B}^{p}, \mathscr{D}_{\alpha}$ is closed under multiplication. Hence, a relevant question is to identify the multiplier algebra of these spaces. Hedenmalm, Lindqvist and Seip identified the multiplier algebra $\mathcal{M}$ of the Hilbert space of Dirichlet series $\mathcal{H}$ proving that

$$
\mathcal{M}=\mathcal{H}^{\infty}
$$

where $\mathcal{H}^{\infty}$ is the algebra of bounded analytic functions on $\mathbb{C}_{0}:=\{s \in \mathbb{C}: \Re(s)>0\}$ which can be represented as a Dirichlet series in some vertical half-plane, [17, Theorem 3.1]. This identification was a key step in solving Beurling's question on complete sequences in $L^{2}(0,1)$. It is noticeable that for all the spaces $\mathcal{H}^{p}, \mathcal{H}_{\alpha}, \mathscr{A}_{\mu}^{p}, \mathscr{B}^{p}, \mathscr{D}_{\alpha}$ the multiplier algebra is also the algebra $\mathcal{H}^{\infty} ;$ [6, Theorem 7], [19, Theorem 1.11], [2, Theorem 10.1 and Theorem 11.21], [3, Theorem 3].

In this paper we consider the space $\mathcal{H}\left(\right.$ ces $\left._{p}\right)$, for $1<p<\infty$, of all Dirichlet series $f(s)=\sum_{n=1}^{\infty} a_{n} n^{-s}$ such that the sequence of coefficients $\left(a_{n}\right)_{n=1}^{\infty}$ belongs to the Cesàro sequence space $c_{p}$. The space ces $_{p}$ consists of all complex sequences whose absolute Cesàro means are in $\ell^{p}$, that is, complex sequences $\left(a_{n}\right)_{n=1}^{\infty}$ satisfying 


$$
\left\|\left(a_{n}\right)_{n=1}^{\infty}\right\|_{\text {ces }_{p}}:=\left(\sum_{n=1}^{\infty}\left(\frac{1}{n} \sum_{k=1}^{n}\left|a_{k}\right|\right)^{p}\right)^{\frac{1}{p}}<\infty .
$$

It is a Banach space of sequences, that arises in a natural way from Hardy's inequality,

$$
\sum_{n=1}^{\infty}\left(\frac{1}{n} \sum_{k=1}^{n}\left|a_{k}\right|\right)^{p} \leq\left(\frac{p}{p-1}\right)^{p} \sum_{n=1}^{\infty}\left|a_{n}\right|^{p}
$$

which establishes the boundedness on $\ell^{p}$ of the Cesàro averaging operator, [15, Theorem 326]. It has been throughly studied by G. Bennett, [8] and Jagers, [18], see also [1] and the references therein.

We define, for $f(s)=\sum_{n=1}^{\infty} a_{n} n^{-s} \in \mathcal{H}\left(c e s_{p}\right)$,

$$
\|f\|_{\mathcal{H}\left(c e s_{p}\right)}:=\left\|\left(a_{n}\right)_{n=1}^{\infty}\right\|_{c e s_{p}} .
$$

With this definition, $\mathcal{H}\left(\right.$ ces $\left._{p}\right)$ is linearly isometric to $c e s_{p}$. The aim of this paper is to study $\mathcal{H}\left(\right.$ ces $\left._{p}\right)$ as a Banach space of analytic functions, to find the maximal common domain of definition of its Dirichlet series, to study the boundedness and the norm of point evaluations, and to identify the algebra of analytic multipliers on $\mathcal{H}\left(c e s_{p}\right)$. As we will see, the situation will turn out to be rather different to that of the previously studied spaces of Dirichlet series.

The paper is organized as follows. Section 2 contains preliminary facts on Dirichlet series and spaces of bounded Dirichlet series.

In Section 3 we study $\mathcal{H}\left(\right.$ ces $\left._{p}\right)$ as a Banach space of analytic functions. From being isometrically isomorphic to $c e s_{p}$, it follows that the sequence of monomials $\left\{m^{-s}: m \geq 1\right\}$ forms an unconditional, boundedly complete and shrinking Schauder basis for $\mathcal{H}\left(\right.$ ces $\left._{p}\right)$; in particular, $\mathcal{H}\left(\right.$ ces $\left._{p}\right)$ is reflexive. We show that all functions in $\mathcal{H}\left(\right.$ ces $\left._{p}\right)$ are analytic on the vertical half-plane $\mathbb{C}_{1 / q}$, where $1 / p+1 / q=1$ (Theorem 3.3). We also study the boundedness on $\mathcal{H}\left(\right.$ ces $\left._{p}\right)$ of point evaluations: $f \mapsto f\left(s_{0}\right)$ for $s_{0} \in \mathbb{C}_{1 / q}$, giving sharp estimates for their norm and the precise order of growth when $\Re(s)$ approaches the critical value $1 / q$ (Theorem 3.4).

Section 4 is devoted to identifying the multiplier algebra $\mathcal{M}\left(\mathcal{H}\left(c e s_{p}\right)\right)$ of $\mathcal{H}\left(c e s_{p}\right)$. A first result shows that

$$
\mathcal{A}^{1 / q} \subseteq \mathcal{M}\left(\mathcal{H}\left(\text { ces }_{p}\right)\right) \varsubsetneqq \mathcal{H}^{\infty}\left(\mathbb{C}_{1 / q}\right),
$$

where $\mathcal{A}^{1 / q}$ is the space of all Dirichlet series $f(s)=\sum_{n=1}^{\infty} a_{n} n^{-s}$ satisfying the condition $\sum_{n=1}^{\infty}\left|a_{n}\right| n^{-1 / q}<$ $\infty$, and $\mathcal{H}^{\infty}\left(\mathbb{C}_{1 / q}\right)$ is the algebra of bounded analytic functions on $\mathbb{C}_{1 / q}$ which can be represented as a Dirichlet series (Theorem 4.5). The result shows that the situation concerning the multiplier algebra of $\mathcal{H}\left(\right.$ ces $\left._{p}\right)$ is completely different from that of other spaces of Dirichlet series studied previously in the literature: in this case, the multiplier algebra will not coincide with an algebra of bounded Dirichlet series.

The fact that the multiplier algebras of $\mathcal{H}^{p}, \mathcal{H}_{\alpha}, \mathscr{A}_{\mu}^{p}, \mathscr{B}^{p}, \mathscr{D}_{\alpha}$ coincide with $\mathcal{H}^{\infty}$ is in accordance withactually, it follows from-the situation of multipliers for Hardy spaces on the unit disc $\mathbb{D}$ of $\mathbb{C}$. More precisely, it follows from the classical result of Schur identifying the multiplier algebra of the Hardy space $H^{2}(\mathbb{D})$, of all Taylor series with square summable coefficients, with the space $H^{\infty}(\mathbb{D})$ of bounded analytic functions on $\mathbb{D},[21, \mathrm{X}$ p. 226].

In the search of a conjecture to pursue, it is relevant to recall the situation regarding multipliers of the space $H\left(\mathbb{D}, c e s_{p}\right)$ of all Taylor series on $\mathbb{D}$ with coefficients belonging to $c e s_{p}$. It was proven by Curbera and Ricker that the multiplier algebra of $H\left(\mathbb{D}, c e s_{p}\right)$ ) is not $H^{\infty}(\mathbb{D})$ but a rather smaller algebra, namely, the Wiener algebra of all absolutely convergent Taylor series, which is the smallest algebra inside $H\left(\mathbb{D}, c e s_{p}\right)$ which contains the polynomials, [12, Theorem 3.1], [13, Theorem 4.1]. 
The main result of this paper is that

$$
\mathcal{M}\left(\mathcal{H}\left(\operatorname{ces}_{p}\right)\right)=\mathcal{A}^{1 / q}
$$

with equality of norms (Theorem 4.8). We attempt an explanation of this unexpected result. Hardy's inequality (1.1) shows that $\ell^{p}$ is continuously included in $c e s_{p}$; in fact, the inclusion is proper. Even more, $c e s_{p}$ contains sequences with arbitrarily large terms. Indeed, given any sequence $\left(a_{k}\right)_{k=1}^{\infty}$ of complex numbers, there exists a subsequence $\left(e^{m_{k}}\right)_{k=1}^{\infty}$ of the canonical vectors $\left\{e^{m}: m \geq 1\right\}$ in $\mathbb{C}^{\mathbb{N}}$ such that $\sum_{k=1}^{\infty} a_{k} e^{m_{k}}$ belongs to $c e s_{p}$. This is an important feature of $c e s_{p}$. Thus, the space $\mathcal{H}\left(\right.$ ces $\left._{p}\right)$ contains Dirichlet series whose coefficients can be arbitrarily large. This feature of $\mathcal{H}\left(c e s_{p}\right)$ may explain the multiplier algebra being the smallest possible algebra which contains the Dirichlet polynomials.

We end in Section 5 with two further results about multipliers on $\mathcal{H}\left(\right.$ ces $\left._{p}\right)$ : regarding compact multipliers (Theorem 5.1) and on the Schur point-wise multipliers from $\mathcal{H}\left(c e s_{p}\right)$ to $\mathcal{A}^{1 / q}$ (Theorem 5.2).

\section{Preliminaries}

We collect some general facts on Dirichlet series. Recall that if a Dirichlet series is convergent (or absolutely convergent) at a point $s_{0} \in \mathbb{C}$, then it is convergent (or absolutely convergent) at any point $s \in \mathbb{C}$ such that $\Re(s)>\Re\left(s_{0}\right)$. As a consequence, convergence regions for Dirichlet series are vertical half-planes $\mathbb{C}_{\sigma}:=\{s \in \mathbb{C}: \Re(s)>\sigma\}$ for $\sigma \in \mathbb{R}$. Given a Dirichlet series $f(s)=\sum_{n=1}^{\infty} a_{n} n^{-s}$, its abscissa of convergence, denoted by $\sigma_{c}(f)$, is the infimum of all $\sigma \in \mathbb{R}$ such that the series converges on the vertical half-plane $\mathbb{C}_{\sigma}$; its abscissa of absolute convergence $\sigma_{a}(f)$ is the infimum of all $\sigma \in \mathbb{R}$ such that the series converges absolutely on $\mathbb{C}_{\sigma}$; and its abscissa of uniform convergence $\sigma_{u}(f)$ is the infimum of all $\sigma \in \mathbb{R}$ such that the series converges uniformly on $\mathbb{C}_{\sigma}$. It follows that $-\infty \leq \sigma_{c}(f) \leq \sigma_{u}(f) \leq \sigma_{a}(f) \leq+\infty$, and $\sigma_{a}(f)-\sigma_{c}(f) \leq 1$ if both values are finite. Bohr proved that $\sigma_{a}(f)-\sigma_{u}(f) \leq 1 / 2$ (the sharpness of this inequality is a celebrated theorem of Bohnenblust and Hille, see [20, Theorem 5.4.2]). There is a further abscissa associated to a Dirichlet series, the abscissa of regularity and boundedness, $\sigma_{b}(f)$, which is the infimum of all $\sigma \in \mathbb{R}$ such that the function $f(s)=\sum_{n=1}^{\infty} a_{n} n^{-s}$ (possibly by analytic continuation from a smaller vertical half-plane) is analytic and bounded on $\mathbb{C}_{\sigma}$. Bohr's theorem assures that $\sigma_{u}(f)=\sigma_{b}(f)$; [10], see also [20, Theorem 6.2.3].

We denote by $\mathcal{D}$ the set of all Dirichlet series which are convergent at some point; this can be equivalently defined as the set of all Dirichlet series such that the sequence of its coefficients has, at most, polynomial growth rate. Given a Banach space of Dirichlet series $\mathcal{E} \subseteq \mathcal{D}$, the abscissa of convergence of $\mathcal{E}$ is defined by $\sigma_{c}(\mathcal{E}):=\sup \left\{\sigma_{c}(f): f \in \mathcal{E}\right\}$, and the abscissa of absolute convergence of $\mathcal{E}$ is $\sigma_{a}(\mathcal{E}):=\sup \left\{\sigma_{a}(f): f \in \mathcal{E}\right\}$. In the case when $\sigma_{c}(\mathcal{E})<\infty$, for every $s_{0} \in \mathbb{C}_{\sigma_{c}(\mathcal{E})}$ it is meaningful to consider the linear functional $\delta_{s_{0}}$ on $\mathcal{E}$ given by point evaluation at $s_{0}$, that is, $f \in \mathcal{E} \mapsto \delta_{s_{0}}(f):=f\left(s_{0}\right) \in \mathbb{C}$.

Throughout the paper we will consider $1<p<\infty$, and $q$ will denote the conjugate exponent of $p$, that is, $1 / p+1 / q=1$.

Further notation used in the paper follows. We denote the set of natural numbers $\{1,2, \ldots\}$ by $\mathbb{N}$. As usual, $\mathbb{R}$ and $\mathbb{C}$ denote the fields of real and complex numbers, respectively. Given a complex number $s \in \mathbb{C}$, its real part is written as $\Re(s)$ and its imaginary part as $\Im(s)$. For $\theta \in \mathbb{R}$, the vertical half-plane at the abscissa $\theta$ is denoted by $\mathbb{C}_{\theta}:=\{s \in \mathbb{C}: \Re(s)>\theta\}$. The unit disc of the complex plane is $\mathbb{D}:=\{z \in \mathbb{C}:|z|<1\}$. For $\Omega$ a region in $\mathbb{C}$, the space of all analytic functions on $\Omega$ will be denoted by $H(\Omega)$. For $k, n \in \mathbb{N}$ we write $k \mid n$ whenever $k$ is a divisor of $n$. The integer part of $x \in \mathbb{R}$, the largest integer which does not exceed $x$, will be denoted by $\lfloor x\rfloor$.

We write $\zeta$ for the Riemann zeta-function, $\zeta(s):=\sum_{n=1}^{\infty} n^{-s}$, for $\Re(s)>1$. The constant function with value one is denoted by 1 . 
Spaces of bounded Dirichlet series play an important role. We collect some relevant facts on them. For $r \in \mathbb{R}$, the space $\mathcal{H}^{\infty}\left(\mathbb{C}_{r}\right)$ consists of all bounded analytic functions on $\mathbb{C}_{r}$ which can be represented as a Dirichlet series in some vertical half-plane, that is,

$$
\mathcal{H}^{\infty}\left(\mathbb{C}_{r}\right):=\mathcal{D} \cap H^{\infty}\left(\mathbb{C}_{r}\right)
$$

Regarding the abscissa of convergence, we have

$$
\sigma_{c}\left(\mathcal{H}^{\infty}\left(\mathbb{C}_{0}\right)\right)=0 \text { and } \sigma_{a}\left(\mathcal{H}^{\infty}\left(\mathbb{C}_{0}\right)\right)=1 / 2
$$

The first statement follows from Bohr's theorem, and the second from a Bohnenblust and Hille's theorem; see, for example, [5, Theorem 1.1.2)]. For $\mathcal{H}^{\infty}\left(\mathbb{C}_{r}\right)$ with $r \neq 0$, consider the translation map $\tau_{r}: \mathcal{D} \rightarrow \mathcal{D}$ given by $\tau_{r}(f)(s):=f(s+r)$, that is,

$$
\tau_{r}\left(\sum_{n=1}^{\infty} a_{n} n^{-s}\right)=\sum_{n=1}^{\infty} a_{n} n^{-(s+r)}=\sum_{n=1}^{\infty}\left(a_{n} n^{-r}\right) n^{-s} .
$$

The translation $\tau_{r}$ establishes an isometric isomorphism between $\mathcal{H}^{\infty}\left(\mathbb{C}_{r}\right)$ and $\mathcal{H}^{\infty}\left(\mathbb{C}_{0}\right)$ from which it follows that

$$
\sigma_{c}\left(\mathcal{H}^{\infty}\left(\mathbb{C}_{r}\right)\right)=r \text { and } \sigma_{a}\left(\mathcal{H}^{\infty}\left(\mathbb{C}_{r}\right)\right)=r+1 / 2
$$

The space $\mathcal{H}^{\infty}\left(\mathbb{C}_{r}\right)$ is a linear space which will be endowed with the supremum norm

$$
\|f\|_{\mathcal{H} \infty\left(\mathbb{C}_{r}\right)}:=\sup _{s \in \mathbb{C}_{r}}|f(s)|, \quad f \in \mathcal{H}^{\infty}\left(\mathbb{C}_{r}\right) .
$$

The isometric isomorphism between $\mathcal{H}^{\infty}\left(\mathbb{C}_{r}\right)$ and $\mathcal{H}^{\infty}\left(\mathbb{C}_{0}\right)$ allows showing the completeness of $\mathcal{H}^{\infty}\left(\mathbb{C}_{r}\right)$ for the supremum norm. The result of Hedenmalm, Lindqvist and Seip states that $\mathcal{H}^{\infty}\left(\mathbb{C}_{0}\right)$ is isometrically isomorphic to the multiplier algebra $\mathcal{M}$ of the Hilbert space of Dirichlet series $\mathcal{H}$, [17, Theorem 3.1]. Since this last space is complete (for the operator norm) it follows that $\mathcal{H}^{\infty}\left(\mathbb{C}_{0}\right)$ is complete for the supremum norm. Hence, $\mathcal{H}^{\infty}\left(\mathbb{C}_{r}\right)$ endowed with the supremum norm is a Banach space.

For issues related to Dirichlet's series we refer the reader to [9], [16], [20], [22, Ch. IX].

\section{The space of Dirichlet series $\mathcal{H}\left(\operatorname{ces}_{p}\right)$}

The space $\mathcal{H}\left(\right.$ ces $\left._{p}\right)$, endowed with the norm (1.2), is a Banach space of Dirichlet series that inherits its functional properties from the sequence space $c e s_{p}$, as $\mathcal{H}\left(c e s_{p}\right)$ and $c e s_{p}$ are linearly isometric. In particular, we have the following result; see [18] and [13, Proposition 2.1].

Proposition 3.1. The following statements hold:

(a) For every $f(s)=\sum_{n=1}^{\infty} a_{n} n^{-s} \in \mathcal{H}\left(\right.$ ces $\left._{p}\right)$ the Dirichlet polynomials $\sum_{n=1}^{N} a_{n} n^{-s}$ converge $($ as $N \rightarrow \infty)$ to $f$ in the norm of $\mathcal{H}\left(\right.$ ces $\left._{p}\right)$. Moreover, from the monotonicity of the norm of ces $s_{p}$,

$$
\|f\|_{\mathcal{H}\left(\operatorname{ces}_{p}\right)}=\sup _{N \in \mathbb{N}}\left\|\sum_{n=1}^{N} a_{n} n^{-s}\right\|_{\mathcal{H}\left(c e s_{p}\right)} .
$$

(b) The sequence of monomials $\left\{m^{-s}: m \geq 1\right\}$ is an unconditional, boundedly complete and shrinking Schauder basis for $\mathcal{H}\left(\right.$ ces $\left._{p}\right)$. In particular, $\mathcal{H}\left(\right.$ ces $\left._{p}\right)$ is reflexive. 
A further approximation for functions in $\mathcal{H}\left(\right.$ ces $\left._{p}\right)$ is possible. Let $\left(p_{k}\right)_{k=1}^{\infty}$ denote the sequence of the prime numbers written in increasing order. For $r \in \mathbb{N}$, let

$$
\mathbb{N}_{r}:=\left\{n \in \mathbb{N}: n=\prod_{i=1}^{r} p_{i}^{t_{i}}, t_{1}, \ldots, t_{r} \geq 0\right\}
$$

Consider the map $Q_{r}$ defined by

$$
f(s)=\sum_{n=1}^{\infty} a_{n} n^{-s} \mapsto Q_{r}(f):=\sum_{n \in \mathbb{N}_{r}} a_{n} n^{-s} .
$$

The map $Q_{r}$ is in fact a projection $Q_{r}: \mathcal{H}\left(c e s_{p}\right) \rightarrow \mathcal{H}\left(c e s_{p}\right)$. A remarkable property of the projection $Q_{r}$ is its multiplicativity, namely, $Q_{r}(f g)=Q_{r}(f) Q_{r}(g)$, which holds for any pair of Dirichlet series $f$ and $g$, see [20, p. 157].

Similarly to Proposition 3.1.(a), the following result holds.

Proposition 3.2. For each $f(s)=\sum_{n=1}^{\infty} a_{n} n^{-s}$ in $\mathcal{H}\left(\right.$ ces $\left._{p}\right)$ the Dirichlet series $\sum_{n \in \mathbb{N}_{r}} a_{n} n^{-s}$ converge (as $r \rightarrow \infty)$ to $f$ in the norm of $\mathcal{H}\left(\right.$ ces $\left._{p}\right)$. Moreover,

$$
\|f\|_{\mathcal{H}\left(c e s_{p}\right)}=\sup _{r \in \mathbb{N}}\left\|\sum_{n \in \mathbb{N}_{r}} a_{n} n^{-s}\right\|_{\mathcal{H}\left(\operatorname{css}_{p}\right)} .
$$

Let us show that $\mathcal{H}\left(c e s_{p}\right)$ is a Banach space of analytic functions. For this, we determine the abscissa of convergence and the abscissa of absolute convergence of $\mathcal{H}\left(\right.$ ces $\left._{p}\right)$.

Theorem 3.3. Every Dirichlet series $f \in \mathcal{H}\left(\right.$ ces $\left._{p}\right)$ converges, in fact absolutely, on the vertical half-plane $\mathbb{C}_{1 / q}$. Moreover, the value $1 / q$ cannot be improved, that is,

$$
\sigma_{c}\left(\mathcal{H}\left(\operatorname{ces}_{p}\right)\right)=\sigma_{a}\left(\mathcal{H}\left(\text { ces }_{p}\right)\right)=1 / q \text {. }
$$

Consequently, $\mathcal{H}\left(\right.$ ces $\left._{p}\right)$ is a Banach space of analytic functions on $\mathbb{C}_{1 / q}$, which is a maximal domain.

Proof. Let $f(s)=\sum_{n=1}^{\infty} a_{n} n^{-s} \in \mathcal{H}\left(c e s_{p}\right)$ with $\left(a_{n}\right)_{n=1}^{\infty} \in c e s_{p}$. Set $r>1 / q$. It follows that

$$
\begin{aligned}
\sum_{n=1}^{\infty} \frac{\left|a_{n}\right|}{n^{r}} & \leq r \sum_{n=1}^{\infty}\left|a_{n}\right| \sum_{k=n}^{\infty} \frac{1}{k^{r+1}}=r \sum_{k=1}^{\infty} \frac{1}{k^{r+1}} \sum_{n=1}^{k}\left|a_{n}\right| \\
& \leq r\left(\sum_{k=1}^{\infty} \frac{1}{k^{r q}}\right)^{1 / q}\left(\sum_{k=1}^{\infty}\left(\frac{1}{k} \sum_{n=1}^{k}\left|a_{n}\right|\right)^{p}\right)^{1 / p} \\
& =r \zeta(r q)^{1 / q}\|f\|_{\mathcal{H}\left(\text { ces }_{p}\right)} .
\end{aligned}
$$

Then $\sigma_{a}(f) \leq 1 / q$ for all $f \in \mathcal{H}\left(\right.$ ces $\left._{p}\right)$ and so $\sigma_{a}\left(\mathcal{H}\left(c e s_{p}\right)\right) \leq 1 / q$.

On the other hand, for $r>1 / p$ set $f(s):=\sum_{n=1}^{\infty} 1 / n^{r+s}$. Note that $f \in \mathcal{H}\left(\right.$ ces $\left._{p}\right)$ as $\left(n^{-r}\right)_{n=1}^{\infty} \in \ell^{p} \subseteq \operatorname{ces}_{p}$. Since $f(s)=\zeta(r+s)$, it follows that $\sigma_{c}(f)=1-r$ which tends to $1 / q$ as $r \rightarrow 1 / p$. Thus, $\sigma_{c}\left(\mathcal{H}\left(\right.\right.$ ces $\left.\left._{p}\right)\right) \geq 1 / q$ and the conclusion follows since $\sigma_{c}\left(\mathcal{H}\left(\right.\right.$ ces $\left.\left._{p}\right)\right) \leq \sigma_{a}\left(\mathcal{H}\left(\right.\right.$ ces $\left.\left._{p}\right)\right)$.

We study next boundedness of the linear functional $\delta_{s_{0}}$ on $\mathcal{H}\left(\right.$ ces $\left._{p}\right)$ given by evaluation at a point $s_{0} \in \mathbb{C}_{1 / q}$ : 


$$
f \in \mathcal{H}\left(\text { ces }_{p}\right) \mapsto \delta_{s_{0}}(f):=f\left(s_{0}\right) \in \mathbb{C} .
$$

Note, for $s_{0}=\sigma+i t \in \mathbb{C}_{1 / q}$ and $f(s)=\sum_{n=1}^{\infty} a_{n} n^{-s} \in \mathcal{H}\left(c e s_{p}\right)$, that the proof of Theorem 3.3 shows

$$
\left|\delta_{s_{0}}(f)\right|=\left|\sum_{n=1}^{\infty} a_{n} n^{-s_{0}}\right| \leq \sum_{n=1}^{\infty}\left|a_{n}\right| n^{-\sigma} \leq \sigma \zeta(\sigma q)^{1 / q}\|f\|_{\mathcal{H}\left(\text { ces }_{p}\right)} .
$$

Thus, $\delta_{s_{0}}$ belongs to the dual space $\mathcal{H}\left(c e s_{p}\right)^{*}$ of $\mathcal{H}\left(c e s_{p}\right)$ with $\left\|\delta_{s_{0}}\right\| \leq \sigma \zeta(\sigma q)^{1 / q}$.

We provide sharp estimates for the norm $\left\|\delta_{s_{0}}\right\|$, the precise order of growth when the abscissa approaches from the right the critical value $1 / q$, and the asymptotic value when the abscissa increases to $\infty$.

We require the dual Banach space of $c s_{p}$. This space was isometrically identified by Jagers, [18]. A simpler isomorphic identification was given by Bennett, [8, p. 61]. Following Bennett the dual space ces $_{p}^{*}$ of $\operatorname{ces}_{p}$ can be identified with the sequence space $d(q)$, for $1 / p+1 / q=1$, of all complex sequences $\left(b_{n}\right)_{n=1}^{\infty}$ satisfying

$$
\left\|\left(b_{n}\right)_{n=1}^{\infty}\right\|_{d(q)}:=\left(\sum_{n=1}^{\infty} \sup _{k \geq n}\left|b_{k}\right|^{q}\right)^{1 / q}<\infty .
$$

The action of a sequence $\left(b_{n}\right)_{n=1}^{\infty} \in d(q)$ as an element of $c e s_{p}^{*}$ is given by the standard pairing

$$
\left(a_{n}\right)_{n=1}^{\infty} \in \operatorname{ces}_{p} \mapsto\left\langle\left(b_{n}\right)_{n=1}^{\infty},\left(a_{n}\right)_{n=1}^{\infty}\right\rangle:=\sum_{n=1}^{\infty} a_{n} b_{n} .
$$

The equivalence between the norms of $c e s_{p}^{*}$ and $d(q)$ is given, for $\left(b_{n}\right)_{n=1}^{\infty} \in c e s_{p}^{*}$, by

$$
\frac{1}{q}\left\|\left(b_{n}\right)_{n=1}^{\infty}\right\|_{d(q)} \leq\left\|\left(b_{n}\right)_{n=1}^{\infty}\right\|_{c e s_{p}^{*}} \leq(p-1)^{1 / p}\left\|\left(b_{n}\right)_{n=1}^{\infty}\right\|_{d(q)}
$$

The sequence $\left(\tilde{b}_{n}\right)_{n=1}^{\infty}$ defined by $\tilde{b}_{n}:=\sup _{k \geq n}\left|b_{k}\right|$, for $n \geq 1$, is known as the least decreasing majorant of the sequence $\left(b_{n}\right)_{n=1}^{\infty}$.

Theorem 3.4. For each $s_{0}=\sigma+i t \in \mathbb{C}_{1 / q}$ the linear functional $\delta_{s_{0}}$ is bounded on $\mathcal{H}\left(\right.$ ces $\left._{p}\right)$, and

$$
\frac{1}{q} \zeta(\sigma q)^{1 / q} \leq\left\|\delta_{s_{0}}\right\| \leq(p-1)^{1 / p} \zeta(\sigma q)^{1 / q}
$$

Moreover, there is a value $\sigma_{p}$, depending only on $p$, such that $\left\|\delta_{s_{0}}\right\|=\zeta(p)^{-1 / p}$ whenever $\sigma \geq \sigma_{p}$.

Proof. Let $s_{0}=\sigma+i t \in \mathbb{C}_{1 / q}$. For $f(s)=\sum_{n=1}^{\infty} a_{n} n^{-s} \in \mathcal{H}\left(c e s_{p}\right)$, since $\left(n^{-s_{0}}\right)_{n=1}^{\infty} \in d(q)$, we can write

$$
\delta_{s_{0}}(f)=f\left(s_{0}\right)=\sum_{n=1}^{\infty} a_{n} n^{-s_{0}}=\left\langle\left(n^{-s_{0}}\right)_{n=1}^{\infty},\left(a_{n}\right)_{n=1}^{\infty}\right\rangle .
$$

Thus, $\delta_{s_{0}}$ acting on $\mathcal{H}\left(\right.$ ces $\left._{p}\right)$ can be identified with the sequence $\left(n^{-s_{0}}\right)_{n=1}^{\infty}$ acting on ces $_{p}$. Since $\mathcal{H}\left(\right.$ ces $\left._{p}\right)$ and $c e s_{p}$ are isometric, we have that the norms of $\delta_{s_{0}}$ as an element of $\mathcal{H}\left(c e s_{p}\right)^{*}$ and of $\left(n^{-s_{0}}\right)_{n=1}^{\infty}$ as an element of $c e s_{p}^{*}$ are equal. Using Bennett's identification of $c e s_{p}^{*}$ as the space $d(q)$, from (3.1), it follows that

$$
\frac{1}{q}\left\|\left(n^{-s_{0}}\right)_{n=1}^{\infty}\right\|_{d(q)} \leq\left\|\delta_{s_{0}}\right\| \leq(p-1)^{1 / p}\left\|\left(n^{-s_{0}}\right)_{n=1}^{\infty}\right\|_{d(q)}
$$


Note that for sequences $\left(b_{n}\right)_{n=1}^{\infty}$ such that the sequence $\left(\left|b_{n}\right|\right)_{n=1}^{\infty}$ is decreasing, we have that $\left(b_{n}\right)_{n=1}^{\infty} \in d(q)$ if and only if $\left(b_{n}\right)_{n=1}^{\infty} \in \ell^{q}$, and in this case the norms coincide. Consequently,

$$
\left\|\left(n^{-s_{0}}\right)_{n=1}^{\infty}\right\|_{d(q)}=\left\|\left(n^{-s_{0}}\right)_{n=1}^{\infty}\right\|_{\ell^{q}}=\left(\sum_{n=1}^{\infty} \frac{1}{n^{\sigma q}}\right)^{1 / q}=\zeta(\sigma q)^{1 / q} .
$$

In order to prove that $\left\|\delta_{s_{0}}\right\|$ becomes constant when $\sigma=\Re\left(s_{0}\right)$ is sufficiently large (only depending on $p$ ) we require the isometric identification of $c e s_{p}^{*}$ given by Jagers, [18]. Namely, for $b=\left(b_{n}\right)_{n=1}^{\infty} \in c e s_{p}^{*}$ we have

$$
\begin{aligned}
\left\|\left(b_{n}\right)_{n=1}^{\infty}\right\|_{c e s_{p}^{*}} & = \\
& \left(\sum_{n \in D(b)}\left(\frac{\left|b_{m(n)}\right|-\left|b_{m(n+1)}\right|}{B_{m(n)}-B_{m(n+1)}}\right)^{q}\left(B_{m(n)}-B_{m(n+1)}\right)\right)^{1 / q},
\end{aligned}
$$

where

$$
\begin{aligned}
& B_{k}:=\sum_{j=k}^{\infty} 1 / j^{p}, \quad k \geq 1 \\
& m(1):=\max \left\{k \in \mathbb{N} \cup\{\infty\}:\left|b_{k}\right|=\max _{j \geq 1}\left|b_{j}\right|\right\},
\end{aligned}
$$

and, for $n \geq 1$,

$$
\begin{aligned}
m(n+1):=\max \{k \in \mathbb{N} \cup\{\infty\}: k>m(n), \\
\left.\frac{\left|b_{m(n)}\right|-\left|b_{k}\right|}{B_{m(n)}-B_{k}}=\min _{m(n)<j \leq \infty} \frac{\left|b_{m(n)}\right|-\left|b_{j}\right|}{B_{m(n)}-B_{j}}\right\},
\end{aligned}
$$

provided $m(n)$ is defined and finite, else $m(n+1)$ is not defined; and $D(b)$ is the set of all $k \geq 1$ such that $m(k)$ is defined and finite. It is understood $b_{\infty}=B_{\infty}=0$.

Note that if $\left(\left|b_{n}\right|\right)_{n=1}^{\infty}$ is strictly decreasing then $m(1)=1$. Moreover, $m(2)=\infty$ if the condition

$$
\frac{\left|b_{1}\right|-\left|b_{n}\right|}{B_{1}-B_{n}} \geq \frac{\left|b_{1}\right|-\left|b_{\infty}\right|}{B_{1}-B_{\infty}}=\frac{\left|b_{1}\right|}{B_{1}}
$$

is satisfied for all $n \geq 2$. In this case $D(b)=\{1\}$ and so $\left\|\left(b_{n}\right)_{n=1}^{\infty}\right\|_{c e s_{p}^{*}}=\left|b_{1}\right| \zeta(p)^{-1 / p}$.

For $b=\left(n^{-s_{0}}\right)_{n=1}^{\infty} \in c e s_{p}^{*}$, we claim that condition (3.3) holds provided that

$$
\sigma \geq \sigma_{p}:=p-1+\frac{\log (p-1)+\log \zeta(p)}{\log 2}
$$

Write (3.3) for this particular sequence:

$$
\frac{1-\frac{1}{n^{\sigma}}}{\sum_{j=1}^{n-1} \frac{1}{j^{p}}} \geq \frac{1}{\sum_{j=1}^{\infty} \frac{1}{j^{p}}}
$$

which is equivalent to

$$
\sum_{j=n}^{\infty} \frac{1}{j^{p}} \geq \frac{1}{n^{\sigma}} \zeta(p)
$$


Since

$$
\sum_{j=n}^{\infty} \frac{1}{j^{p}} \geq \frac{1}{p-1} \cdot \frac{1}{n^{p-1}}
$$

it suffices to prove that

$$
\frac{1}{p-1} \cdot \frac{1}{n^{p-1}} \geq \frac{1}{n^{\sigma}} \zeta(p)
$$

holds for all $n \geq 2$. We rewrite this condition as

$$
n^{\sigma-p+1} \geq(p-1) \zeta(p)
$$

It is clear that for the above inequality to hold, necessarily we must have $\sigma \geq p-1$. In this case, the sequence $\left(n^{\sigma-p+1}\right)_{n=1}^{\infty}$ is increasing. Thus, it suffices to check the above inequality for $n=2$ :

$$
2^{\sigma-p+1} \geq(p-1) \zeta(p),
$$

that is,

$$
\sigma \geq p-1+\frac{\log (p-1)+\log \zeta(p)}{\log 2}=\sigma_{p}
$$

Therefore, for $b=\left(n^{-s_{0}}\right)_{n=1}^{\infty}$ with $s_{0} \in \mathbb{C}_{\sigma_{p}}$, we have that $m(2)=\infty$ and so $D(b)=\{1\}$. Hence the sum in (3.2) has only one term and

$$
\left\|\delta_{s_{0}}\right\|=\left\|\left(n^{-s_{0}}\right)_{n=1}^{\infty}\right\|_{c e s_{p}^{*}}=\zeta(p)^{-1 / p}
$$

Remark 3.5. From the proof of Theorem 3.3 and Theorem 3.4 actually we have, for $s_{0}=\sigma+i t \in \mathbb{C}_{1 / q}$, that

$$
\left\|\delta_{s_{0}}\right\| \leq \min \left\{\sigma,(p-1)^{1 / p}\right\} \zeta(\sigma q)^{1 / q}
$$

Since $1 / q<(p-1)^{1 / p}$, as the function $x \mapsto x^{x}$ is increasing on $(1, \infty)$, we have that

$$
\min \left\{\sigma,(p-1)^{1 / p}\right\}=\left\{\begin{array}{ccc}
\sigma & \text { for } & 1 / q<\sigma \leq(p-1)^{1 / p} \\
(p-1)^{1 / p} & \text { for } & \sigma>(p-1)^{1 / p}
\end{array}\right.
$$

The bounds on the norm of point evaluations in Theorem 3.4 and Remark 3.5 can be sharpened for $\mathcal{H}\left(\mathrm{ces}_{2}\right)$.

Proposition 3.6. Let $1 / 2<\Re\left(s_{0}\right)=\sigma \leq 1$ and $\delta_{s_{0}}: \mathcal{H}\left(\right.$ ces $\left._{2}\right) \rightarrow \mathbb{C}$ be the corresponding point evaluation functional. Then its norm can be written as

$$
\left\|\delta_{s_{0}}\right\|=\left(\sum_{n=1}^{\infty} n^{2}\left(\frac{1}{n^{\sigma}}-\frac{1}{(n+1)^{\sigma}}\right)^{2}\right)^{1 / 2}
$$

and the following bounds hold

$$
\left(2^{\sigma}-1\right) \sqrt{\zeta(2 \sigma)-1} \leq\left\|\delta_{s_{0}}\right\| \leq \sigma \sqrt{\zeta(2 \sigma)-1} .
$$


Proof. We use the isometric identification of ces $_{p}^{*}$ by Jagers for $p=2$.

Let $b=\left(n^{-s_{0}}\right)_{n=1}^{\infty}$. We will prove that in this case, and for every $m \in \mathbb{N}$, the sequence

$$
\left(\frac{\left|b_{m}\right|-\left|b_{n}\right|}{B_{m}-B_{n}}\right)_{n=m+1}^{\infty}
$$

is strictly increasing. This condition is precisely

$$
\frac{\frac{1}{m^{\sigma}}-\frac{1}{n^{\sigma}}}{\sum_{k=m}^{\infty} \frac{1}{k^{2}}-\sum_{k=n}^{\infty} \frac{1}{k^{2}}}<\frac{\frac{1}{m^{\sigma}}-\frac{1}{(n+1)^{\sigma}}}{\sum_{k=m}^{\infty} \frac{1}{k^{2}}-\sum_{k=n+1}^{\infty} \frac{1}{k^{2}}}
$$

which is equivalent to

$$
\frac{\frac{1}{m^{\sigma}}-\frac{1}{n^{\sigma}}}{n^{2}\left(\frac{1}{n^{\sigma}}-\frac{1}{(n+1)^{\sigma}}\right)}<\sum_{k=m}^{n-1} \frac{1}{k^{2}}
$$

By applying the mean value theorem to the function $f(x)=x^{\sigma}$ on $(m, n)$ and $(n, n+1)$ we obtain, for $1 / 2<\sigma \leq 1$, that

$$
\frac{\frac{1}{m^{\sigma}}-\frac{1}{n^{\sigma}}}{n^{2}\left(\frac{1}{n^{\sigma}}-\frac{1}{(n+1)^{\sigma}}\right)}=\frac{(n+1)^{\sigma}\left(n^{\sigma}-m^{\sigma}\right)}{n^{2} m^{\sigma}\left((n+1)^{\sigma}-n^{\sigma}\right)} \leq \frac{n+1}{n^{2} m}(n-m) .
$$

In order to bound the right-hand side of (3.5) we use the following inequality:

$$
\int_{m}^{n} \frac{d x}{x^{2}}+\frac{1}{2}\left(\frac{1}{m^{2}}-\frac{1}{n^{2}}\right) \leq \sum_{k=m}^{n-1} \frac{1}{k^{2}}
$$

see for instance [14, p. 54]. Since

$$
\int_{m}^{n} \frac{d x}{x^{2}}+\frac{1}{2}\left(\frac{1}{m^{2}}-\frac{1}{n^{2}}\right)=\left(\frac{1}{m}-\frac{1}{n}\right)\left(1+\frac{1}{2 m}+\frac{1}{2 n}\right)
$$

we have

$$
\left(\frac{n-m}{m n}\right)\left(1+\frac{1}{2 m}+\frac{1}{2 n}\right) \leq \sum_{k=m}^{n-1} \frac{1}{k^{2}}
$$

Then, (3.6) and (3.7) reduce the validity of (3.5) to

$$
\frac{n+1}{n^{2} m}(n-m)<\left(\frac{n-m}{m n}\right)\left(1+\frac{1}{2 m}+\frac{1}{2 n}\right)
$$

which is true since $m<n$. Thus, (3.5) holds and so, for every $m \in \mathbb{N}$, the sequence (3.4) is strictly increasing. 
Hence, for each $n \in \mathbb{N}$ we have that $m(n)=n$. This implies that $D(b)=\mathbb{N}$ for $b=\left(n^{-s_{0}}\right)_{n=1}^{\infty}$ and so

$$
\left\|\delta_{s_{0}}\right\|=\left\|\left(n^{-s_{0}}\right)_{n=1}^{\infty}\right\|_{c e s_{2}^{*}}=\left(\sum_{n=1}^{\infty} n^{2}\left(\frac{1}{n^{\sigma}}-\frac{1}{(n+1)^{\sigma}}\right)^{2}\right)^{1 / 2} .
$$

Since

$$
n\left(\frac{1}{n^{\sigma}}-\frac{1}{(n+1)^{\sigma}}\right)=\frac{1}{(n+1)^{\sigma}} g\left(n^{-1}\right)
$$

where $g(x)=\frac{(1+x)^{\sigma}-1}{x}$ decreases in $(0, \infty)$, we have

$$
\frac{2^{\sigma}-1}{(n+1)^{\sigma}} \leq n\left(\frac{1}{n^{\sigma}}-\frac{1}{(n+1)^{\sigma}}\right) \leq \frac{\sigma}{(n+1)^{\sigma}}
$$

and so the bounds for $\left\|\delta_{s_{0}}\right\|$ follow.

In the case $p=2$, there are two equivalent expressions for the norm in ces $_{2}$ (and so for the norm in $\mathcal{H}\left(\right.$ ces $\left.\left._{2}\right)\right)$ which are of independent interest.

Proposition 3.7. Let $a=\left(a_{n}\right)_{n=1}^{\infty} \in$ ces $_{2}$. Define the functionals

$$
\begin{aligned}
& M(a):=\left(\sum_{i, j=1}^{\infty} \frac{\left|a_{i}\right|\left|a_{j}\right|}{\max \{i, j\}}\right)^{1 / 2} \\
& N(a):=\left(\sum_{n=1}^{\infty} \frac{\left|a_{n}\right|}{n} \sum_{k=1}^{n}\left|a_{k}\right|\right)^{1 / 2} .
\end{aligned}
$$

Then

$$
N(a) \leq M(a) \leq\|a\|_{\text {ces }_{2}} \leq \sqrt{2} M(a) \leq 2 N(a) .
$$

Proof. Rearranging the sums in the norm of $a$ we obtain that

$$
\begin{aligned}
\|a\|_{c e s_{2}}^{2} & =\sum_{n=1}^{\infty}\left(\frac{1}{n} \sum_{k=1}^{n}\left|a_{k}\right|\right)^{2} \\
& =\sum_{n=1}^{\infty} \frac{1}{n^{2}}\left(\sum_{1 \leq i, j \leq n}\left|a_{i}\right|\left|a_{j}\right|\right) \\
& =\sum_{i, j=1}^{\infty}\left|a_{i} \| a_{j}\right|\left(\sum_{n \geq i, j} \frac{1}{n^{2}}\right) .
\end{aligned}
$$

Since $1 / n \leq \sum_{k=n}^{\infty} k^{-2} \leq 2 / n$ for every $n \geq 1$, it follows that

$$
\frac{1}{\max \{i, j\}} \leq \sum_{n \geq i, j} \frac{1}{n^{2}} \leq \frac{2}{\max \{i, j\}}
$$

Hence, we deduce that $M(a) \leq\|a\|_{\text {ces }_{2}} \leq \sqrt{2} M(a)$. 
On the other hand,

$$
\begin{aligned}
M(a)^{2} & =\sum_{i, j=1}^{\infty} \frac{\left|a_{i}\right|\left|a_{j}\right|}{\max \{i, j\}}=\sum_{n=1}^{\infty} \frac{1}{n}\left(\sum_{\max \{i, j\}=n}\left|a_{i}\right|\left|a_{j}\right|\right) \\
& =\sum_{n=1}^{\infty} \frac{\left|a_{n}\right|}{n}\left(\left|a_{n}\right|+2 \sum_{k=1}^{n-1}\left|a_{k}\right|\right) \\
& \leq 2 \sum_{n=1}^{\infty} \frac{\left|a_{n}\right|}{n} \sum_{k=1}^{n}\left|a_{k}\right|=2 N(a)^{2} .
\end{aligned}
$$

In a similar way

$$
M(a)^{2} \geq \sum_{n=1}^{\infty} \frac{\left|a_{n}\right|}{n} \sum_{k=1}^{n}\left|a_{k}\right|=N(a)^{2} .
$$

Consequently, $N(a) \leq M(a) \leq \sqrt{2} N(a)$.

\section{The multiplier algebra of $\mathcal{H}\left(\right.$ ces $\left._{p}\right)$}

Given a Banach space of Dirichlet series $\mathcal{E} \subseteq \mathcal{D}$ with convergence abscissa $\sigma_{c}(\mathcal{E})$, a multiplier on $\mathcal{E}$ is an analytic function $f$ on $\mathbb{C}_{\sigma_{c}(\mathcal{E})}$ with the property that $f g \in \mathcal{E}$ for every $g \in \mathcal{E}$. The multiplier algebra of $\mathcal{E}$ is the space of all multipliers on $\mathcal{E}$, which will be denoted by $\mathcal{M}(\mathcal{E})$. Standard arguments give the following facts on $\mathcal{M}(\mathcal{E})$.

Proposition 4.1. Let $\mathcal{E} \subseteq \mathcal{D}$ be a Banach space of Dirichlet series. Suppose that there exists $\sigma \geq \sigma_{c}(\mathcal{E})$ such that the point evaluation functional $\delta_{s_{0}}$ is continuous on $\mathcal{E}$ for every $s_{0} \in \mathbb{C}_{\sigma}$. Then the following holds:

(a) For every $f \in \mathcal{M}(\mathcal{E})$, the operator $M_{f}: \mathcal{E} \rightarrow \mathcal{E}$, given by $M_{f}(g):=$ fg for all $g \in \mathcal{E}$, is linear and bounded.

(b) If the constant function $\mathbf{1} \in \mathcal{E}$, then $\mathcal{M}(\mathcal{E}) \subseteq \mathcal{E}$ and for every $f \in \mathcal{M}(\mathcal{E})$ it follows that $\|f\|_{\mathcal{E}} \leq$ $\|\mathbf{1}\|_{\mathcal{E}}\|f\|_{\mathcal{M}(\mathcal{E})}$, where $\|f\|_{\mathcal{M}(\mathcal{E})}$ denotes the operator norm of $M_{f}$. Moreover, in this case $\mathcal{M}(\mathcal{E})$ is a closed subspace of the space $B(\mathcal{E})$ of all bounded linear operators of $\mathcal{E}$ into itself, and, endowing $\mathcal{M}(\mathcal{E})$ with the norm $\|\cdot\|_{\mathcal{M}(\mathcal{E})}$, the inclusion $\mathcal{M}(\mathcal{E}) \subseteq \mathcal{E}$ is continuous with embedding constant equal to $\|\mathbf{1}\|_{\mathcal{E}}$.

The next proposition shows that, under minimal conditions which guarantee a good behavior of $\mathcal{M}(\mathcal{E})$, every multiplier on $\mathcal{E}$ is a bounded analytic function on the appropriate domain.

Proposition 4.2. Let $\mathcal{E} \subseteq \mathcal{D}$ be a Banach space of Dirichlet series satisfying the condition of Proposition 4.1 for some $\sigma \geq \sigma_{c}(\mathcal{E})$ and such that $\mathbf{1} \in \mathcal{E}$. Then,

$$
\mathcal{M}(\mathcal{E}) \subseteq \mathcal{H}^{\infty}\left(\mathbb{C}_{\sigma}\right)
$$

where the inclusion is continuous with continuity constant equal to one.

Proof. Let $f \in \mathcal{M}(\mathcal{E})$. By Proposition 4.1, we have that $\mathcal{M}(\mathcal{E}) \subseteq \mathcal{E}$ and so $f^{2}=f f \in \mathcal{E}$ with

$$
\left\|f^{2}\right\|_{\mathcal{E}} \leq\|f\|_{\mathcal{E}}\|f\|_{\mathcal{M}(\mathcal{E})} \leq\|\mathbf{1}\|_{\mathcal{E}}\|f\|_{\mathcal{M}(\mathcal{E})}^{2}
$$

Iterating the above procedure, we obtain, for every $n \geq 1$, that $f^{n} \in \mathcal{E}$ and 


$$
\left\|f^{n}\right\|_{\mathcal{E}} \leq\|\mathbf{1}\|_{\mathcal{E}}\|f\|_{\mathcal{M}(\mathcal{E})}^{n} .
$$

For each $s_{0} \in \mathbb{C}_{\sigma}$, by hypothesis, the point evaluation functional $\delta_{s_{0}}$ is bounded on $\mathcal{E}$. Then

$$
\left|f^{n}\left(s_{0}\right)\right|=\left|\delta_{s_{0}}\left(f^{n}\right)\right| \leq\left\|\delta_{s_{0}}\right\| \cdot\left\|f^{n}\right\|_{\mathcal{E}} \leq\left\|\delta_{s_{0}}\right\| \cdot\|\mathbf{1}\|_{\mathcal{E}}\|f\|_{\mathcal{M}(\mathcal{E})}^{n}
$$

Since $\left|f^{n}\left(s_{0}\right)\right|=\left|f\left(s_{0}\right)\right|^{n}$, it follows that

$$
\left|f\left(s_{0}\right)\right| \leq\left(\left\|\delta_{s_{0}}\right\| \cdot\|\mathbf{1}\|_{\mathcal{E}}\right)^{1 / n}\|f\|_{\mathcal{M}(\mathcal{E})} .
$$

Making $n \rightarrow \infty$ we have that $\left|f\left(s_{0}\right)\right| \leq\|f\|_{\mathcal{M}(\mathcal{E})}$. Hence, $f \in \mathcal{H}^{\infty}\left(\mathbb{C}_{\sigma}\right)$ and $\|f\|_{\mathcal{H}}\left(\mathbb{C}_{\sigma}\right) \leq\|f\|_{\mathcal{M}(\mathcal{E})}$.

Furthermore, if the monomials $n^{-s}$, for $n \geq 1$, are multipliers on $\mathcal{E}$, a certain natural weighted $\ell^{1}$-space of Dirichlet series is included in $\mathcal{M}(\mathcal{E})$.

Proposition 4.3. Let $\mathcal{E} \subseteq \mathcal{D}$ be a Banach space of Dirichlet series satisfying the condition of Proposition 4.1 for some $\sigma \geq \sigma_{c}(\mathcal{E})$ and such that $\mathbf{1} \in \mathcal{E}$. Suppose that $\left\{n^{-s}: n \geq 1\right\} \subset \mathcal{M}(\mathcal{E})$ and denote $\mu_{n}:=\left\|n^{-s}\right\|_{\mathcal{M}(\mathcal{E})}$ for $n \geq 1$. Then

$$
\mathcal{A}\left(\left(\mu_{n}\right)_{n=1}^{\infty}\right):=\left\{f(s)=\sum_{n=1}^{\infty} a_{n} n^{-s}: \sum_{n=1}^{\infty}\left|a_{n}\right| \mu_{n}<\infty\right\} \subseteq \mathcal{M}(\mathcal{E})
$$

and $\|f\|_{\mathcal{M}(\mathcal{E})} \leq \sum_{n=1}^{\infty}\left|a_{n}\right| \mu_{n}$ for all $f \in \mathcal{A}\left(\left(\mu_{n}\right)_{n=1}^{\infty}\right)$.

Proof. Let $f(s)=\sum_{n=1}^{\infty} a_{n} n^{-s} \in \mathcal{A}\left(\left(\mu_{n}\right)_{n=1}^{\infty}\right)$. The series $\sum_{n=1}^{\infty} a_{n} n^{-s}$ is absolutely convergent in $\mathcal{M}(\mathcal{E})$, as

$$
\sum_{n=1}^{\infty}\left\|a_{n} n^{-s}\right\|_{\mathcal{M}(\mathcal{E})}=\sum_{n=1}^{\infty}\left|a_{n}\right| \mu_{n}<\infty
$$

and so it converges in norm to some $h \in \mathcal{M}(\mathcal{E})$. Since, $\mathcal{M}(\mathcal{E}) \subseteq \mathcal{E}$ continuously and so norm convergence in $\mathcal{M}(\mathcal{E})$ implies pointwise convergence on $\mathbb{C}_{\sigma}$, it follows that $f=h \in \mathcal{M}(\mathcal{E})$. From the equality above it follows that $\|f\|_{\mathcal{M}(\mathcal{E})} \leq \sum_{n=1}^{\infty}\left|a_{n}\right| \mu_{n}$.

Remark 4.4. The particular spaces $\mathcal{A}\left(\left(\mu_{n}\right)_{n=1}^{\infty}\right)$ above obtained for $r \in \mathbb{R}$ and $\mu_{n}:=n^{-r}$ for all $n \geq 1$, are denoted by

$$
\mathcal{A}^{r}:=\left\{f(s)=\sum_{n=1}^{\infty} a_{n} n^{-s}: \sum_{n=1}^{\infty}\left|a_{n}\right| n^{-r}<\infty\right\} .
$$

They are Banach spaces when endowed with the norm $\|f\|_{\mathcal{A}^{r}}:=\sum_{n=1}^{\infty}\left|a_{n}\right| n^{-r}$. When $r=0$, the corresponding space is the well known Wiener-Dirichlet algebra $\mathcal{A}^{+}$, see [7]. Direct computation shows that $\sigma_{c}\left(\mathcal{A}^{r}\right)=\sigma_{a}\left(\mathcal{A}^{r}\right)=r$. Regarding the point evaluations on $\mathcal{A}^{r}$, we have that $\left\|\delta_{s_{0}}\right\|=1$, for every $s_{0} \in \mathbb{C}_{r}$. With respect to the multipliers, by Proposition $4.1, \mathcal{M}\left(\mathcal{A}^{r}\right) \subseteq \mathcal{A}^{r}$ continuously with embedding constant equal to one. In fact, both spaces coincide with equality of norms. To see this, we check that monomials are multiplier on $\mathcal{A}^{r}$. For $m \in \mathbb{N}$, consider $m^{-s}$ and let $g(s)=\sum_{n=1}^{\infty} a_{n} n^{-s} \in \mathcal{A}^{r}$. Noting that $m^{-s} g(s)=\sum_{n=1}^{\infty} c_{n} n^{-s}$ with $c_{n}=a_{\frac{n}{m}}$ if $m \mid n$ and $c_{n}=0$ in other case, it follows

$$
\sum_{n=1}^{\infty}\left|c_{n}\right| n^{-r}=\sum_{\substack{n=1 \\ m \mid n}}^{\infty}\left|a_{\frac{n}{m}}\right| n^{-r}=\sum_{k=1}^{\infty}\left|a_{k}\right|(k m)^{-r}=m^{-r}\|g\|_{\mathcal{A}^{r}}
$$


and so $m^{-s} g \in \mathcal{A}^{r}$ with $\left\|m^{-s} g\right\|_{\mathcal{A}^{r}}=m^{-r}\|g\|_{\mathcal{A}^{r}}$. Hence, $m^{-s} \in \mathcal{M}\left(\mathcal{A}^{r}\right)$ and $\left\|m^{-s}\right\|_{\mathcal{M}\left(\mathcal{A}^{r}\right)} \leq m^{-r}$. Actually $\left\|m^{-s}\right\|_{\mathcal{M}\left(\mathcal{A}^{r}\right)}=m^{-r}$, as $\mathbf{1} \in \mathcal{A}^{r}$ with norm one and $\left\|m^{-s}\right\|_{\mathcal{A}^{r}}=m^{-r}$. Therefore, from Proposition 4.3 , the inclusion $\mathcal{A}^{r} \subseteq \mathcal{M}\left(\mathcal{A}^{r}\right)$ holds continuously with embedding constant equal to one.

We now consider the space $\mathcal{M}\left(\mathcal{H}\left(c e s_{p}\right)\right)$ of all multipliers on $\mathcal{H}\left(\right.$ ces $\left._{p}\right)$. We have seen that $\sigma_{c}\left(\mathcal{H}\left(c e s_{p}\right)\right)=$ $1 / q$ and that, for each $s_{0} \in \mathbb{C}_{1 / q}$, the point evaluation functional $\delta_{s_{0}}$ is continuous on $\mathcal{H}\left(\right.$ ces $\left._{p}\right)$, see Theorem 3.3 and Theorem 3.4. With these conditions, Proposition 4.1 implies that every multiplier $f$ on $\mathcal{H}\left(\right.$ ces $\left._{p}\right)$ defines a bounded multiplication operator $M_{f}$ from $\mathcal{H}\left(c e s_{p}\right)$ into itself:

$$
g \in \mathcal{H}\left(\text { ces }_{p}\right) \mapsto M_{f}(g)=f g \in \mathcal{H}\left(\text { ces }_{p}\right) .
$$

Moreover, since the constant function $\mathbf{1} \in \mathcal{H}\left(\operatorname{ces}_{p}\right)$ and $\|\mathbf{1}\|_{\mathcal{H}\left(\operatorname{ces}_{p}\right)}=\zeta(p)^{1 / p}$, it also follows that

$$
\mathcal{M}\left(\mathcal{H}\left(c e s_{p}\right)\right) \subseteq \mathcal{H}\left(\operatorname{ces}_{p}\right)
$$

continuously with embedding constant $\zeta(p)^{1 / p}$, that is,

$$
\|f\|_{\mathcal{H}\left(c e s_{p}\right)} \leq \zeta(p)^{1 / p} \cdot\|f\|_{\mathcal{M}\left(\mathcal{H}\left(\operatorname{ces}_{p}\right)\right)}, \quad f \in \mathcal{M}\left(\mathcal{H}\left(\operatorname{ces}_{p}\right)\right)
$$

So, a multiplier $f$ on $\mathcal{H}\left(c e s_{p}\right)$ is actually a Dirichlet series $f(s)=\sum_{n=1}^{\infty} a_{n} n^{-s}$ belonging to $\mathcal{H}\left(\right.$ ces $\left._{p}\right)$ and the action of the multiplication operator $M_{f}$ on $g(s)=\sum_{n=1}^{\infty} b_{n} n^{-s} \in \mathcal{H}\left(\right.$ ces $\left._{p}\right)$ is given by

$$
M_{f}(g)(s)=f(s) g(s)=\sum_{n=1}^{\infty}\left(\sum_{k \mid n} a_{k} b_{\frac{n}{k}}\right) n^{-s} .
$$

The boundedness of the operator $M_{f}$ corresponds to the existence of some constant $M>0$ such that

$$
\left(\sum_{n=1}^{\infty}\left(\frac{1}{n} \sum_{k=1}^{n}\left|\sum_{j \mid k} a_{j} b_{\frac{k}{j}}\right|\right)^{p}\right)^{1 / p} \leq M \cdot\left(\sum_{n=1}^{\infty}\left(\frac{1}{n} \sum_{k=1}^{n}\left|b_{k}\right|\right)^{p}\right)^{1 / p}
$$

for all $g(s)=\sum_{n=1}^{\infty} b_{n} n^{-s} \in \mathcal{H}\left(c e s_{p}\right)$. The least of such constants $M$ is the operator norm $\left\|M_{f}\right\|$ of $M_{f}$ as a bounded operator from $\mathcal{H}\left(\right.$ ces $\left._{p}\right)$ into itself, which we denote by $\|f\|_{\mathcal{M}\left(\mathcal{H}\left(\operatorname{ces}_{p}\right)\right)}$.

Theorem 4.5. The inclusions

$$
\mathcal{A}^{1 / q} \subseteq \mathcal{M}\left(\mathcal{H}\left(\text { ces }_{p}\right)\right) \varsubsetneqq \mathcal{H}^{\infty}\left(\mathbb{C}_{1 / q}\right)
$$

holds continuously with inclusion constants equal to one.

Proof. The first inclusion follows from Proposition 4.3 if we show, for $m \geq 1$, that the monomial $m^{-s}$ is a multiplier on $\mathcal{H}\left(c e s_{p}\right)$, and

$$
\left\|m^{-s}\right\|_{\mathcal{M}\left(\mathcal{H}\left(\operatorname{ces}_{p}\right)\right)}=m^{-1 / q}
$$

Let $g(s)=\sum_{n=1}^{\infty} b_{n} n^{-s} \in \mathcal{H}\left(c e s_{p}\right)$. The coefficients of the Dirichlet series $m^{-s} g$ are given by

$$
\left(m^{-s} \cdot g\right)_{k}=\left\{\begin{array}{ll}
b_{i} & \text { if } k=i m \text { for some } i \geq 1 \\
0 & \text { in other case }
\end{array} .\right.
$$


We estimate:

$$
\begin{aligned}
\left\|m^{-s} g\right\|_{\mathcal{H}\left(\text { ces } s_{p}\right)}^{p} & =\sum_{n=1}^{\infty}\left(\frac{1}{n} \sum_{k=1}^{n}\left|\left(m^{-s} \cdot g\right)_{k}\right|\right)^{p}=\sum_{n=m}^{\infty}\left(\frac{1}{n} \sum_{i=1}^{\lfloor n / m\rfloor}\left|b_{i}\right|\right)^{p} \\
& =\sum_{j=1}^{\infty} \sum_{n=j m}^{(j+1) m-1}\left(\frac{1}{n} \sum_{i=1}^{j}\left|b_{i}\right|\right)^{p}=\sum_{j=1}^{\infty}\left(\sum_{i=1}^{j}\left|b_{i}\right|\right)^{p} \sum_{n=j m}^{(j+1) m-1} \frac{1}{n^{p}} \\
& \leq \sum_{j=1}^{\infty}\left(\sum_{i=1}^{j}\left|b_{i}\right|\right)^{p} \frac{m}{(j m)^{p}}=\frac{1}{m^{p-1}}\|g\|_{\mathcal{H}\left(\text { ces }_{p}\right)}^{p} .
\end{aligned}
$$

Then $\left\|m^{-s} g\right\|_{\mathcal{H}\left(\text { ces }_{p}\right)} \leq m^{-1 / q}\|g\|_{\mathcal{H}\left(\text { ces }_{p}\right)}$. Thus, $m^{-s}$ is a multiplier on $\mathcal{H}\left(\right.$ ces $\left._{p}\right)$ and $\left\|m^{-s}\right\|_{\mathcal{M}\left(\mathcal{H}\left(\text { ces }_{p}\right)\right)} \leq$ $m^{-1 / q}$.

On the other hand, for $g(s)=j^{-s}$, with $j \geq 2$, we have

$$
\left\|m^{-s} j^{-s}\right\|_{\mathcal{H}\left(\text { ces }_{p}\right)}^{p}=\sum_{n=j m}^{\infty} \frac{1}{n^{p}} \geq \frac{1}{p-1} \frac{1}{(j m)^{p-1}}
$$

and

$$
\left\|j^{-s}\right\|_{\mathcal{H}\left(\text { ces }_{p}\right)}^{p}=\sum_{n=j}^{\infty} \frac{1}{n^{p}} \leq \frac{1}{p-1} \frac{1}{(j-1)^{p-1}} .
$$

Hence,

$$
\left\|m^{-s}\right\|_{\mathcal{M}\left(\mathcal{H}\left(\operatorname{ces}_{p}\right)\right)} \geq \frac{\left\|m^{-s} \cdot j^{-s}\right\|_{\mathcal{H}\left(\operatorname{ces}_{p}\right)}}{\left\|j^{-s}\right\|_{\mathcal{H}\left(\operatorname{ces}_{p}\right)}} \geq \frac{(j-1)^{1 / q}}{(j m)^{1 / q}} .
$$

Making $j \rightarrow \infty$, we arrive at $\left\|m^{-s}\right\|_{\mathcal{M}\left(\mathcal{H}\left(\text { ces }_{p}\right)\right)} \geq m^{-1 / q}$.

The second inclusion follows from Proposition 4.2.

It only remains to prove that $\mathcal{M}\left(\mathcal{H}\left(\right.\right.$ ces $\left.\left._{p}\right)\right) \neq \mathcal{H}^{\infty}\left(\mathbb{C}_{1 / q}\right)$. For this we calculate the abscissa of convergence and absolute convergence of $\mathcal{M}\left(\mathcal{H}\left(\right.\right.$ ces $\left.\left._{p}\right)\right)$. From Theorem 3.3, Remark 4.4 and the inclusions

$$
\mathcal{A}^{1 / q} \subseteq \mathcal{M}\left(\mathcal{H}\left(\operatorname{ces}_{p}\right)\right) \subseteq \mathcal{H}\left(\operatorname{ces}_{p}\right),
$$

it follows that

$$
\begin{aligned}
1 / q=\sigma_{c}\left(\mathcal{A}^{1 / q}\right) & \leq \sigma_{c}\left(\mathcal{M}\left(\mathcal{H}\left(\text { ces }_{p}\right)\right)\right) \\
& \leq \sigma_{a}\left(\mathcal{M}\left(\mathcal{H}\left(\text { ces }_{p}\right)\right)\right) \leq \sigma_{a}\left(\mathcal{H}\left(\text { ces }_{p}\right)\right)=1 / q
\end{aligned}
$$

Then $\sigma_{c}\left(\mathcal{M}\left(\mathcal{H}\left(\right.\right.\right.$ ces $\left.\left.\left._{p}\right)\right)\right)=\sigma_{a}\left(\mathcal{M}\left(\mathcal{H}\left(\right.\right.\right.$ ces $\left.\left.\left._{p}\right)\right)\right)=1 / q$. Thus, $\mathcal{M}\left(\mathcal{H}\left(\right.\right.$ ces $\left.\left._{p}\right)\right) \neq \mathcal{H}^{\infty}\left(\mathbb{C}_{1 / q}\right)$ as $\sigma_{a}\left(\mathcal{H}^{\infty}\left(\mathbb{C}_{1 / q}\right)\right)=$ $1 / q+1 / 2$ by $(2.1)$.

Theorem 4.5 already shows that the situation concerning the multiplier algebra of $\mathcal{H}\left(\right.$ ces $\left._{p}\right)$ is certainly different from that of other spaces of Dirichlet series studied previously in the literature. In this case, the multiplier algebra will not coincide with an algebra of bounded Dirichlet series. Next we will prove that

$$
\mathcal{M}\left(\mathcal{H}\left(\operatorname{ces}_{p}\right)\right)=\mathcal{A}^{1 / q},
$$


with equality of norms. As explained in the Introduction, this fact is, to some extent, analogous to the case of the space $H\left(\mathbb{D}, c e s_{p}\right)$, of Taylor series on the unit disc $\mathbb{D}$ of the complex plane with coefficients belonging to $c e s_{p}$, in which case its multiplier algebra is the Wiener algebra of absolutely convergent Taylor series, which is the smallest algebra inside $H\left(\mathbb{D}, c_{p}\right)$ containing the polynomials.

The proof of (4.1), which will be given in Theorem 4.8, is rather technical. We first discuss the strategy of the proof in order to help its better understanding. Given $f(s)=\sum_{n=1}^{\infty} a_{n} n^{-s} \in \mathcal{M}\left(\mathcal{H}\left(\right.\right.$ ces $\left.\left._{p}\right)\right)$, for adequate values of the parameter $\alpha$, we find a sequence $\left(g^{m, \alpha}\right)_{m=1}^{\infty}$ in $\mathcal{H}\left(c e s_{p}\right)$ such that

$$
\sum_{n=1}^{\infty}\left|a_{n}\right| n^{-1 / q}=\lim _{\substack{\alpha \rightarrow 1 / q \\ m \rightarrow \infty}} \frac{\left\|f g^{m, \alpha}\right\|_{\mathcal{H}\left(\text { ces }_{p}\right)}}{\left\|g^{m, \alpha}\right\|_{\mathcal{H}\left(\text { ces }_{p}\right)}} \leq\|f\|_{\mathcal{M}\left(\mathcal{H}\left(\text { ces }_{p}\right)\right)} .
$$

Estimating the norm of $f g^{m, \alpha}$ in $\mathcal{H}\left(c^{2} s_{p}\right)$ is complicated since it requires, apart from the Cesàro means, dealing with the coefficients of the product of two Dirichlet series. With the aim of having these coefficients as simple as possible, we consider functions $g^{m, \alpha}(s)=\sum_{n=1}^{\infty} b_{n}^{m, \alpha} n^{-s}$ whose coefficients are supported on certain subsets of the prime numbers $\left(p_{r}\right)_{r=1}^{\infty}$. For an adequate sequence $\left(r_{m}\right)_{m=2}^{\infty} \subseteq \mathbb{N}$, we require that $b_{n}^{m, \alpha} \neq 0$ only when $n=p_{r}$ for $r \geq r_{m}$. The key point is that, for coefficients having index of the form $k=\omega p_{r}$ with $r \geq r_{m}$ and $\omega=\prod_{i=1}^{r_{m}-1} p_{i}^{t_{i}}, t_{1}, \ldots, t_{r_{m}-1} \geq 0$, the corresponding coefficient of the product $f g^{m, \alpha}$ is reduced to one term

$$
\sum_{j \mid k} a_{j} b_{\frac{k}{j}}^{m, \alpha}=a_{\omega} b_{p_{r}}^{m, \alpha}
$$

In this way, estimating the norms $\left\|f g^{m, \alpha}\right\|_{\mathcal{H}\left(\text { ces }_{p}\right)}$ and $\left\|g^{m, \alpha}\right\|_{\mathcal{H}\left(\text { ces }_{p}\right)}$ is reduced to estimating sums of the form

$$
\sum_{\substack{r \geq r_{m} \\ p_{r} \leq \gamma}} b_{p_{r}}^{m, \alpha}
$$

where $\gamma \in\left[r_{m}, \infty\right)$; note that the summation is taken over the set $\left\{r \in \mathbb{N}: r \geq r_{m}\right.$ and $\left.p_{r} \leq \gamma\right\}$. To this end, we consider the function $\phi(x):=x \log x$ on $[1, \infty)$ and choose, via the Prime Number Theorem, $\left(r_{m}\right)_{m=2}^{\infty} \subseteq \mathbb{N}$ such that $p_{r}$ is sufficiently close to $\phi(r)$ for $r \geq r_{m}$. The problem is then transformed into estimating sums of the form

$$
\sum_{r=r_{m}}^{\phi^{-1}(\gamma)} b_{p_{r}}^{m, \alpha}
$$

Finally, good estimates for the above sum are obtained by taking $b_{p_{r}}^{m, \alpha}=\left(\phi^{\alpha}\right)^{\prime}(r)$.

We require two lemmata.

Lemma 4.6. Let $0<\beta<1$ and $\phi(x)=x \log x$ for $x \in[1, \infty)$. There exists $x_{\beta}$ such that for every $r_{0} \in \mathbb{N}$ with $r_{0} \geq x_{\beta}, C_{1} \geq C_{2} \geq \phi\left(r_{0}\right)$, and $J$ satisfying

$$
\left\{r \in \mathbb{N}: r \geq r_{0} \text { and } \phi(r) \leq C_{2}\right\} \subset J \subset\left\{r \in \mathbb{N}: r \geq r_{0} \text { and } \phi(r) \leq C_{1}\right\}
$$

it follows that

$$
C_{2}^{\alpha}-\phi\left(r_{0}\right)^{\alpha} \leq \sum_{r \in J}\left(\phi^{\alpha}\right)^{\prime}(r) \leq C_{1}^{\alpha}-\phi\left(r_{0}-1\right)^{\alpha}
$$

for all $\alpha \leq \beta$. 
Proof. We consider the Lambert function $W$ on $(0, \infty)$ defined by $W(x) e^{W(x)}=x$; see [11]. Then

$$
\phi\left(\frac{x}{W(x)}\right)=x .
$$

Let $r_{0} \in \mathbb{N}, C_{1} \geq C_{2} \geq \phi\left(r_{0}\right)$, and $J$ satisfy (4.2). By (4.4) and since $\phi$ is increasing and injective on $[1, \infty)$, we have that $r \leq \frac{x}{W(x)}$ if and only if $\phi(r) \leq \phi\left(\frac{x}{W(x)}\right)=x$. Then, it follows that

$$
\sum_{r=r_{0}}^{\left\lfloor\frac{C_{2}}{W\left(C_{2}\right)}\right\rfloor} h(r) \leq \sum_{r \in J} h(r) \leq \sum_{r=r_{0}}^{\left\lfloor\frac{C_{1}}{W\left(C_{1}\right)}\right\rfloor} h(r)
$$

for every positive function $h$. For $\alpha \leq \beta$ take $h$ as the derivative of $\phi^{\alpha}$, that is,

$$
h(x)=\left(\phi^{\alpha}\right)^{\prime}(x)=\alpha(x \log x)^{\alpha-1}(\log x+1) .
$$

Let $x_{\beta}$ be sufficiently large so that $h$ is decreasing on $\left[x_{\beta}-1, \infty\right)$. Such value $x_{\beta}$ exists as

$$
\begin{aligned}
h^{\prime}(x) & =\alpha(\alpha-1)(x \log x)^{\alpha-2}(\log x+1)^{2}+\alpha(x \log x)^{\alpha-1} \frac{1}{x} \\
& =\alpha(x \log x)^{\alpha-2}(\log x+1)^{2}\left(\alpha-1+\frac{\log x}{(\log x+1)^{2}}\right) \\
& \leq \alpha(x \log x)^{\alpha-2}(\log x+1)^{2}\left(\beta-1+\frac{\log x}{(\log x+1)^{2}}\right) .
\end{aligned}
$$

Since $\lim _{x \rightarrow \infty} \log x(\log x+1)^{-2}=0$ and $\beta-1<0$, there exists $x_{\beta}$ such that $h^{\prime}(x) \leq 0$ for all $x \geq x_{\beta}-1$.

Then, for every $M \geq N \geq x_{\beta}$ it follows that

$$
\sum_{r=N}^{M} h(r) \leq \sum_{r=N_{r-1}}^{M} \int_{N-1}^{r} h(x) d x=\int_{N}^{M} h(x) d x=\phi(M)^{\alpha}-\phi(N-1)^{\alpha}
$$

and

$$
\sum_{r=N}^{M} h(r) \geq \sum_{r=N}^{M} \int_{r}^{r+1} h(x) d x=\int_{N}^{M+1} h(x) d x=\phi(M+1)^{\alpha}-\phi(N)^{\alpha} .
$$

Hence, from (4.5), if $r_{0} \geq x_{\beta}$ we have

$$
\phi\left(\left\lfloor\frac{C_{2}}{W\left(C_{2}\right)}\right\rfloor+1\right)^{\alpha}-\phi\left(r_{0}\right)^{\alpha} \leq \sum_{r \in J} h(r) \leq \phi\left(\left\lfloor\frac{C_{1}}{W\left(C_{1}\right)}\right\rfloor\right)^{\alpha}-\phi\left(r_{0}-1\right)^{\alpha} .
$$

From (4.4),

$$
\phi\left(\left\lfloor\frac{C_{1}}{W\left(C_{1}\right)}\right\rfloor\right) \leq \phi\left(\frac{C_{1}}{W\left(C_{1}\right)}\right)=C_{1}
$$

and

$$
\phi\left(\left\lfloor\frac{C_{2}}{W\left(C_{2}\right)}\right\rfloor+1\right) \geq \phi\left(\frac{C_{2}}{W\left(C_{2}\right)}\right)=C_{2},
$$

and so (4.3) holds. 
Recall that $p_{r}$ denotes the $r$-th prime number. The Prime Number Theorem

$$
\lim _{r \rightarrow \infty} \frac{p_{r}}{r \log r}=1
$$

allows to find, for each $2 \leq m \in \mathbb{N}$, an integer $r_{m} \in \mathbb{N}$, with $r_{m}>m$ and such that

$$
1-\frac{1}{m} \leq \frac{p_{r}}{r \log r} \leq 1+\frac{1}{m}, \text { for all } r \geq r_{m}
$$

Consequently, we have $\left(r_{m}\right)_{m=2}^{\infty} \subseteq \mathbb{N}$ such that

$$
\frac{m p_{r}}{m+1} \leq r \log r \leq \frac{m p_{r}}{m-1}, \quad \text { for all } r \geq r_{m}
$$

Lemma 4.7. Let $\phi(x)=x \log x$ for $x \in[1, \infty)$ and consider the sequence $\left(r_{m}\right)_{m=2}^{\infty}$ given in (4.6). For $1<q<\infty$, there exists $x_{q}$ such that

$$
\max \left\{\left(\frac{m \gamma}{m+1}\right)^{\alpha}-\phi\left(r_{m}\right)^{\alpha}, 0\right\} \leq \sum_{\substack{r \geq r_{m} \\ p_{r} \leq \gamma}}\left(\phi^{\alpha}\right)^{\prime}(r) \leq\left(\frac{m \gamma}{m-1}\right)^{\alpha}-\phi\left(r_{m}-1\right)^{\alpha}
$$

whenever $r_{m} \geq x_{q}, \gamma \geq p_{r_{m}}$ and $\alpha \leq \frac{1}{q}$.

Proof. Let $\gamma \geq p_{r_{m}}$ and $\alpha \leq \frac{1}{q}$. We apply Lemma 4.6 with $\beta=\frac{1}{q}, r_{0}=r_{m}, C_{1}=\frac{m \gamma}{m-1}, C_{2}=\frac{m \gamma}{m+1}$ if $\gamma \geq \phi\left(r_{m}\right) \frac{m+1}{m}$, and $C_{2}=\phi\left(r_{m}\right)$ in other case, and

$$
J=\left\{r \in \mathbb{N}: r \geq r_{m} \text { and } p_{r} \leq \gamma\right\}
$$

We verify that the hypothesis of Lemma 4.6 hold. By (4.6) we have $\phi\left(r_{m}\right) \leq \frac{m p_{r_{m}}}{m-1} \leq C_{1}$ and so $C_{1} \geq C_{2} \geq \phi\left(r_{m}\right)$. The right-hand inclusion in (4.2) holds since for every $r \in J$, by (4.6), we have that $\phi(r) \leq \frac{m p_{r}}{m-1} \leq C_{1}$. On the other hand, let $r \geq r_{m}$ such that $\phi(r) \leq C_{2}$. If $C_{2}=\frac{m \gamma}{m+1}$, from (4.6) we have that $p_{r} \leq \phi(r) \frac{m+1}{m} \leq C_{2} \frac{m+1}{m}=\gamma$ and so $r \in J$. If $C_{2}=\phi\left(r_{m}\right)$ then $r=r_{m} \in J$. So, the left-hand inclusion of (4.2) holds.

Noting that $C_{2}^{\alpha}-\phi\left(r_{m}\right)^{\alpha}=\max \left\{\left(\frac{m \gamma}{m+1}\right)^{\alpha}-\phi\left(r_{m}\right)^{\alpha}, 0\right\}$, the conclusion follows.

Now we prove the main result.

Theorem 4.8. For $1<p<\infty$ and $1 / p+1 / q=1$, we have

$$
\mathcal{M}\left(\mathcal{H}\left(c e s_{p}\right)\right)=\mathcal{A}^{1 / q}
$$

with equality of norms.

Proof. Let $f(s)=\sum_{n=1}^{\infty} a_{n} n^{-s} \in \mathcal{M}\left(\mathcal{H}\left(\right.\right.$ ces $\left.\left._{p}\right)\right)$ and set $a:=\left(a_{n}\right)_{n=1}^{\infty}$. Take $\phi(x)=x \log x$ for $x \in[1, \infty)$, the sequence $\left(r_{m}\right)_{m=2}^{\infty}$ given in (4.6) and the value $x_{q}$ provided by Lemma 4.7. For fixed $2 \leq m \in \mathbb{N}$ with $r_{m} \geq x_{q}$ and $1 /(2 q)<\alpha<1 / q$, consider the sequence $b^{m, \alpha}=\left(b_{n}^{m, \alpha}\right)_{n=1}^{\infty}$ defined by

$$
b_{n}^{m, \alpha}:= \begin{cases}\left(\phi^{\alpha}\right)^{\prime}(r) & \text { if } n=p_{r} \text { with } r \geq r_{m} \\ 0 & \text { in other case }\end{cases}
$$

Let $g^{m, \alpha}(s):=\sum_{n=1}^{\infty} b_{n}^{m, \alpha} n^{-s}$. Then, from Lemma 4.7, 


$$
\begin{aligned}
\left\|g^{m, \alpha}\right\|_{\mathcal{H}\left(\text { ces }_{p}\right)}^{p}=\left\|b^{m, \alpha}\right\|_{\text {ces }_{p}}^{p} & =\sum_{n=1}^{\infty} \frac{1}{n^{p}}\left(\sum_{k=1}^{n}\left|b_{k}^{m, \alpha}\right|\right)^{p} \\
& =\sum_{n=p_{r_{m}}}^{\infty} \frac{1}{n^{p}}\left(\sum_{\substack{r \geq r_{m} \\
p_{r} \leq n}}\left(\phi^{\alpha}\right)^{\prime}(r)\right)^{p} \\
& \leq \sum_{n=p_{r_{m}}}^{\infty} \frac{1}{n^{p}}\left(\frac{m n}{m-1}\right)^{\alpha p} \\
& =\left(\frac{m}{m-1}\right)^{\alpha p} \sum_{n=p_{r_{m}}}^{\infty} \frac{1}{n^{p(1-\alpha)}} \\
& \leq\left(\frac{m}{m-1}\right)^{\alpha p} \frac{1}{(p(1-\alpha)-1)\left(p_{r_{m}}-1\right)^{p(1-\alpha)-1}} .
\end{aligned}
$$

We estimate $\left\|f g^{m, \alpha}\right\|_{\mathcal{H}\left(\text { ces }_{p}\right)}^{p}$ from below. Note that for each $k=\omega p_{r}$ with $r \geq r_{m}$ and $\omega=\prod_{i=1}^{r_{m}-1} p_{i}^{t_{i}}$, $t_{1}, \ldots, t_{r_{m}-1} \geq 0$, it follows that

$$
\left(a \cdot b^{m, \alpha}\right)_{k}=\sum_{j \mid k} a_{j} b_{\frac{k}{j}}^{m, \alpha}=a_{\omega} b_{p_{r}}^{m, \alpha}=a_{\omega}\left(\phi^{\alpha}\right)^{\prime}(r) .
$$

Indeed, if $j \mid k$ and $j \neq \omega$ we have that $k / j \neq p_{\hat{r}}$ for all $\hat{r} \geq r_{m}$ and so $b_{k / j}^{m, \alpha}=0$. Consider the subset of $\mathbb{N}$ given by

$$
\mathcal{P}_{m}:=\left\{n \in \mathbb{N}: n=\prod_{i=1}^{r_{m}-1} p_{i}^{t_{i}}, 0 \leq t_{i} \leq m \text { for all } 1 \leq i \leq r_{m}-1\right\}
$$

Since $\omega p_{r}=\hat{\omega} p_{\hat{r}}$ with $\omega, \hat{\omega} \in \mathcal{P}_{m}$ and $r, \hat{r} \geq r_{m}$ implies that $\omega=\hat{\omega}$ and $p_{r}=p_{\hat{r}}$, it follows that the set

$$
\mathcal{O}=\bigcup_{\omega \in \mathcal{P}_{m}} \omega \cdot\left\{p_{r}: r \geq r_{m}\right\}
$$

is a finite union of disjoint sets. Then, for any $n_{m} \geq 3 p_{r_{m}}^{m r_{m}+1}$, we have that

$$
\begin{aligned}
\left\|f g^{m, \alpha}\right\|_{\mathcal{H}\left(\text { ces }_{p}\right)}^{p}=\left\|a \cdot b^{m, \alpha}\right\|_{\text {ces }_{p}}^{p} & =\sum_{n=1}^{\infty} \frac{1}{n^{p}}\left(\sum_{k=1}^{n}\left|\left(a \cdot b^{m, \alpha}\right)_{k}\right|\right)^{p} \\
& \geq \sum_{n=n_{m}}^{\infty} \frac{1}{n^{p}}\left(\sum_{\substack{k=1 \\
k \in \mathcal{O}}}^{n}\left|\left(a \cdot b^{m, \alpha}\right)_{k}\right|\right)^{p} \\
& =\sum_{n=n_{m}}^{\infty} \frac{1}{n^{p}}\left(\sum_{\omega \in \mathcal{P}_{m}} \sum_{\substack{r \geq r_{m} \\
p_{r} \leq \frac{n}{\omega}}}\left|\left(a \cdot b^{m, \alpha}\right)_{\omega} p_{r}\right|\right)^{p} \\
& =\sum_{n=n_{m}}^{\infty} \frac{1}{n^{p}}\left(\sum_{\omega \in \mathcal{P}_{m}}\left|a_{\omega}\right| \sum_{\substack{r \geq r_{m} \\
p_{r} \leq \frac{n}{\omega}}}\left(\phi^{\alpha}\right)^{\prime}(r)\right)^{p}
\end{aligned}
$$

Note that $\omega \leq p_{r_{m}}^{m r_{m}}$ whenever $\omega \in \mathcal{P}_{m}$ and so $n / \omega \geq 3 p_{r_{m}}$ for $n \geq n_{m}$. Hence, by (4.6),

$$
\phi\left(r_{m}\right) \leq \frac{m p_{r_{m}}}{m-1} \leq \frac{m n}{3(m-1) \omega} \leq \frac{m n}{(m+1) \omega}
$$


for every $n \geq n_{m}$ and $\omega \in \mathcal{P}_{m}$. Applying Lemma 4.7, it follows that

$$
\left\|f g^{m, \alpha}\right\|_{\mathcal{H}\left(\text { ces }_{p}\right)}^{p} \geq \sum_{n=n_{m}}^{\infty} \frac{1}{n^{p}}\left(\sum_{\omega \in \mathcal{P}_{m}}\left|a_{\omega}\right|\left(\left(\frac{m n}{(m+1) \omega}\right)^{\alpha}-\phi\left(r_{m}\right)^{\alpha}\right)\right)^{p} .
$$

Note that if we restrict to $n_{m} \geq 3 p_{r_{m}}^{m r_{m}+1+2 q}$ we obtain

$$
\begin{aligned}
\left(\frac{m n}{(m+1) \omega}\right)^{\alpha}-\phi\left(r_{m}\right)^{\alpha} & =\left(\frac{m n}{(m+1) \omega}\right)^{\alpha}\left(1-\left(\frac{(m+1) \omega \phi\left(r_{m}\right)}{m n}\right)^{\alpha}\right) \\
& \geq\left(\frac{m n}{(m+1) \omega}\right)^{\alpha}\left(1-\frac{1}{p_{r_{m}}}\right)
\end{aligned}
$$

for every $\omega \in \mathcal{P}_{m}$ and $n \geq n_{m}$. Indeed, by (4.6),

$$
\begin{aligned}
\frac{(m+1) \omega \phi\left(r_{m}\right)}{m} & \leq \frac{(m+1) \omega m p_{r_{m}}}{m(m-1)} \\
& \leq \frac{(m+1) p_{r_{m}}^{m r_{m}+1}}{m-1} \\
& \leq \frac{(m+1) n_{m}}{3(m-1) p_{r_{m}}^{2 q}} \leq \frac{n}{p_{r_{m}}^{1 / \alpha}}
\end{aligned}
$$

where we use that $\alpha>1 /(2 q)$. Then,

$$
\begin{aligned}
\left\|f g^{m, \alpha}\right\|_{\mathcal{H}\left(\text { ces }_{p}\right)}^{p} & \geq \sum_{n=n_{m}}^{\infty} \frac{1}{n^{p}}\left(\sum_{\omega \in \mathcal{P}_{m}}\left|a_{\omega}\right|\left(\frac{m n}{(m+1) \omega}\right)^{\alpha}\left(1-\frac{1}{p_{r_{m}}}\right)\right)^{p} \\
& =\left(1-\frac{1}{p_{r_{m}}}\right)^{p}\left(\frac{m}{m+1}\right)^{\alpha p} \sum_{n=n_{m}}^{\infty} \frac{1}{n^{p(1-\alpha)}}\left(\sum_{\omega \in \mathcal{P}_{m}} \frac{\left|a_{\omega}\right|}{\omega^{\alpha}}\right)^{p} \\
& \geq\left(1-\frac{1}{p_{r_{m}}}\right)^{p}\left(\frac{m}{m+1}\right)^{\alpha p} \frac{1}{(p(1-\alpha)-1) n_{m}^{p(1-\alpha)-1}}\left(\sum_{\omega \in \mathcal{P}_{m}} \frac{\left|a_{\omega}\right|}{\omega^{\alpha}}\right)^{p} .
\end{aligned}
$$

From (4.7) and (4.8) it follows that

$$
\begin{aligned}
\|f\|_{\mathcal{M}\left(\mathcal{H}\left(\text { ces }_{p}\right)\right)}^{p} & \geq \frac{\left\|f g^{m, \alpha}\right\|_{\mathcal{H}\left(\text { ces }_{p}\right)}^{p}}{\left\|g^{m, \alpha}\right\|_{\mathcal{H}\left(\text { ces }_{p}\right)}^{p}} \\
& \geq \frac{\left(1-\frac{1}{p_{r_{m}}}\right)^{p}\left(\frac{m}{m+1}\right)^{\alpha p} \frac{1}{\left(\frac{m}{m-1}\right)^{\alpha p}} \frac{1}{(p(1-\alpha)-1) n_{m}^{p(1-\alpha)-1}}\left(\sum_{\omega \in \mathcal{P}_{m}} \frac{\left|a_{\omega}\right|}{\omega^{\alpha}}\right)^{p}}{\left(\frac{m-1}{m+1}\right)^{\alpha p} \frac{1}{n_{m}^{p(1-\alpha)-1}}\left(\sum_{\omega \in \mathcal{P}_{m}} \frac{\left|a_{\omega}\right|}{\omega^{\alpha}}\right)^{p}} \\
& =\frac{\left(p_{r_{m}}-1\right)^{p(2-\alpha)-1}}{p_{r_{m}}^{p}} .
\end{aligned}
$$

Taking limit as $\alpha \rightarrow 1 / q$ we have

$$
\|f\|_{\mathcal{M}\left(\mathcal{H}\left(c e s_{p}\right)\right)}^{p} \geq \frac{\left(p_{r_{m}}-1\right)^{p}}{p_{r_{m}}^{p}}\left(\frac{m-1}{m+1}\right)^{p / q}\left(\sum_{\omega \in \mathcal{P}_{m}} \frac{\left|a_{\omega}\right|}{\omega^{1 / q}}\right)^{p} .
$$


Finally, making $m \rightarrow \infty$ we conclude

$$
\|f\|_{\mathcal{M}\left(\mathcal{H}\left(\operatorname{ces}_{p}\right)\right)}^{p} \geq\left(\sum_{\omega \in \mathbb{N}} \frac{\left|a_{\omega}\right|}{\omega^{1 / q}}\right)^{p}
$$

\section{Further facts on multipliers on $\mathcal{H}\left(\right.$ ces $\left._{p}\right)$}

First we study the compactness of the multipliers on $\mathcal{H}\left(\right.$ ces $\left._{p}\right)$. It turns out that there is no other compact multiplier than zero.

Theorem 5.1. Let $f \in \mathcal{M}\left(\mathcal{H}\left(\right.\right.$ ces $\left.\left._{p}\right)\right)$. Suppose that the associated operator

$$
g \in \mathcal{H}\left(\operatorname{ces}_{p}\right) \mapsto M_{f}(g):=f g \in \mathcal{H}\left(\operatorname{ces}_{p}\right)
$$

is compact. Then $f=0$.

Proof. Consider the sequence $\left\{m^{1 / q} m^{-s}\right\}_{m=1}^{\infty}$ in $\mathcal{H}\left(c e s_{p}\right)$. It is bounded as, for $m \geq 2$, we have that

$$
\left\|m^{1 / q} m^{-s}\right\|_{\mathcal{H}\left(\text { ces }_{p}\right)}=m^{1 / q}\left\|e^{m}\right\|_{\text {ces }_{p}}=m^{1 / q}\left(\sum_{n=m}^{\infty} \frac{1}{n^{p}}\right)^{1 / p} \leq \frac{2^{1 / q}}{(p-1)^{1 / p}} .
$$

Then, by compactness of $M_{f}$, there exists a subsequence $\left\{m_{k}^{1 / q} m_{k}^{-s}\right\}_{k=1}^{\infty}$ such that $\left\{M_{f}\left(m_{k}^{1 / q} m_{k}^{-s}\right)\right\}_{k=1}^{\infty}$ converges in norm to some $g \in \mathcal{H}\left(c e s_{p}\right)$. For $s_{0} \in \mathbb{C}_{1 / q}$, since the point evaluation $\delta_{s_{0}}$ is bounded on $\mathcal{H}\left(c e s_{p}\right)$, we have

$$
\delta_{s_{0}}\left(M_{f}\left(m_{k}^{1 / q} m_{k}^{-s}\right)\right) \underset{k \rightarrow \infty}{\longrightarrow} \delta_{s_{0}}(g)=g\left(s_{0}\right) .
$$

On the other hand,

$$
\delta_{s_{0}}\left(M_{f}\left(m_{k}^{1 / q} m_{k}^{-s}\right)\right)=f\left(s_{0}\right) m_{k}^{1 / q-s_{0}} \underset{k \rightarrow \infty}{\longrightarrow} 0
$$

Thus, $g=0$. Hence, $\left\{M_{f}\left(m_{k}^{1 / q} m_{k}^{-s}\right)\right\}_{k=1}^{\infty}$ converges to zero in the norm of $\mathcal{H}\left(c e s_{p}\right)$.

We estimate from below $\left\|M_{f}\left(m_{k}^{1 / q} m_{k}^{-s}\right)\right\|_{\mathcal{H}\left(c e s_{p}\right)}=m_{k}^{1 / q}\left\|M_{f}\left(m_{k}^{-s}\right)\right\|_{\mathcal{H}\left(c e s_{p}\right)}$. Let $f(s)=\sum_{n=1}^{\infty} a_{n} n^{-s}$. We have seen in the proof of Theorem 4.5 that

$$
\left\|M_{f}\left(m^{-s}\right)\right\|_{\mathcal{H}\left(\operatorname{ces}_{p}\right)}^{p}=\left\|m^{-s} f\right\|_{\mathcal{H}\left(\operatorname{ces}_{p}\right)}^{p}=\sum_{j=1}^{\infty}\left(\sum_{i=1}^{j}\left|a_{i}\right|\right)^{p} \sum_{n=j m}^{(j+1) m-1} \frac{1}{n^{p}}
$$

Since

$$
\sum_{n=j m}^{(j+1) m-1} \frac{1}{n^{p}} \geq \frac{m}{((j+1) m-1)^{p}} \geq \frac{m}{(2 j m)^{p}}
$$

it follows that

$$
\left\|M_{f}\left(m^{-s}\right)\right\|_{\mathcal{H}\left(\operatorname{ces}_{p}\right)}^{p} \geq \frac{m}{(2 m)^{p}} \sum_{j=1}^{\infty} \frac{1}{j^{p}}\left(\sum_{i=1}^{j}\left|a_{i}\right|\right)^{p}=\frac{m}{(2 m)^{p}}\|f\|_{\mathcal{H}\left(\operatorname{ces}_{p}\right)}^{p} .
$$


Then,

$$
\left\|M_{f}\left(m_{k}^{1 / q} m_{k}^{-s}\right)\right\|_{\mathcal{H}\left(\text { ces }_{p}\right)} \geq m_{k}^{1 / q} \frac{m_{k}^{1 / p}}{2 m_{k}}\|f\|_{\mathcal{H}\left(\text { ces }_{p}\right)}=\frac{1}{2}\|f\|_{\mathcal{H}\left(\text { ces }_{p}\right)} .
$$

Taking $k \rightarrow \infty$ we have that $\|f\|_{\mathcal{H}\left(\text { ces }_{p}\right)} \leq 0$ and so, $f=0$.

Next we discuss how "close" is the space $\mathcal{H}\left(\right.$ ces $\left._{p}\right)$ to its multiplier algebra. Let us first note that $\mathcal{M}\left(\mathcal{H}\left(\right.\right.$ ces $\left.\left._{p}\right)\right)=\mathcal{A}^{1 / q} \varsubsetneqq \mathcal{H}\left(c e s_{p}\right)$. Indeed, in other case the point evaluation $\delta_{1 / q}$ at the point $s_{0}=1 / q$, which belongs to the dual space of $\mathcal{A}^{1 / q}$, would belong to $\mathcal{H}\left(c e s_{p}\right)^{*}$. But this is not true as $\delta_{1 / q} \in \mathcal{H}\left(\text { ces }_{p}\right)^{*}$ is precisely $\left(n^{-1 / q}\right)_{n=1}^{\infty} \in d(q)$ (see the proof of Theorem 3.4), which corresponds to $\left(n^{-1 / q}\right)_{n=1}^{\infty} \in \ell^{q}$.

The multiplier algebra $\mathcal{M}\left(\mathcal{H}\left(\right.\right.$ ces $\left.\left._{p}\right)\right)$ is "close" to $\mathcal{H}\left(\right.$ ces $\left._{p}\right)$ in the sense shown by the following example. For $f(s)=\sum_{n=1}^{\infty} a_{n} n^{-s} \in \mathcal{H}\left(\right.$ ces $\left._{p}\right)$ and $\varepsilon>0$, set

$$
f_{\varepsilon}(s):=\sum_{n=1}^{\infty} \frac{a_{n} n^{-\varepsilon}}{n^{s}} .
$$

Theorem 3.3 shows that $\sigma_{a}\left(\mathcal{H}\left(\right.\right.$ ces $\left.\left._{p}\right)\right)=1 / q$. Then

$$
\sum_{n=1}^{\infty} \frac{\left|a_{n} n^{-\varepsilon}\right|}{n^{1 / q}}=\sum_{n=1}^{\infty} \frac{\left|a_{n}\right|}{n^{1 / q+\epsilon}}<\infty
$$

Consequently, $f_{\varepsilon} \in \mathcal{A}^{1 / q}=\mathcal{M}\left(\mathcal{H}\left(\right.\right.$ ces $\left.\left._{p}\right)\right)$. The question arises: for which sequences $\left(b_{n}\right)_{n=1}^{\infty}$ it is the case that $\sum_{n=1}^{\infty} a_{n} b_{n} n^{-s} \in \mathcal{M}\left(\mathcal{H}\left(c e s_{p}\right)\right)$ whenever $\sum_{n=1}^{\infty} a_{n} n^{-s} \in \mathcal{H}\left(c e s_{p}\right)$ ? Recall that these sequences are called the Schur multipliers between $\mathcal{H}\left(\right.$ ces $\left._{p}\right)$ and $\mathcal{A}^{1 / q}$.

Theorem 5.2. A sequence $\left(b_{n}\right)_{n=1}^{\infty}$ satisfies that for every $\sum_{n=1}^{\infty} a_{n} n^{-s} \in \mathcal{H}\left(c e s_{p}\right)$ the series $\sum_{n=1}^{\infty} a_{n} b_{n} n^{-s}$ is a multiplier on $\mathcal{H}\left(\right.$ cesp $\left._{p}\right)$ if and only if

$$
\left(b_{n} n^{-1 / q}\right)_{n=1}^{\infty} \in d(q)
$$

where $d(q)$ is the dual space of $\mathrm{ces}_{p}$, that is, the following condition holds

$$
\sum_{n=1}^{\infty} \sup _{k \geq n}\left(\frac{\left|b_{k}\right|^{q}}{k}\right)<\infty
$$

Proof. Denote by $\ell_{q}^{1}$ the Banach space of complex sequences $a=\left(a_{n}\right)_{n=1}^{\infty}$ such that $\|a\|_{\ell_{q}^{1}}:=$ $\sum_{n=1}^{\infty}\left|a_{n}\right| n^{-1 / q}<\infty$. The sequence $b=\left(b_{n}\right)_{n=1}^{\infty}$ being a Schur multiplier between $\mathcal{H}\left(\right.$ ces $\left._{p}\right)$ and $\mathcal{A}^{1 / q}$ corresponds precisely to the operator $T_{b}$, defined by

$$
a=\left(a_{n}\right)_{n=1}^{\infty} \in \operatorname{ces}_{p} \mapsto T_{b}(a):=\left(a_{n} b_{n}\right)_{n=1}^{\infty} \in \ell_{q}^{1},
$$

being well defined and, via the closed graph theorem, bounded.

Suppose that $T_{b}$ is well defined. For every $a=\left(a_{n}\right)_{n=1}^{\infty} \in c e s_{p}$ we have that

$$
\left|\left\langle\left(b_{n} n^{-1 / q}\right)_{n=1}^{\infty},\left(a_{n}\right)_{n=1}^{\infty}\right\rangle\right| \leq \sum_{n=1}^{\infty} \frac{\left|a_{n} b_{n}\right|}{n^{1 / q}}=\left\|T_{b}(a)\right\|_{\ell_{q}^{1}} \leq\left\|T_{b}\right\| \cdot\|a\|_{\text {ces }_{p}}
$$

and so $\left(b_{n} n^{-1 / q}\right)_{n=1}^{\infty} \in c e s_{p}^{*}=d(q)$. 
Conversely, suppose that $\left(b_{n} n^{-1 / q}\right)_{n=1}^{\infty} \in c e s_{p}^{*}=d(q)$. For every $a=\left(a_{n}\right)_{n=1}^{\infty} \in c e s_{p}$ we have that

$$
\left|\sum_{n=1}^{\infty} \frac{a_{n} b_{n}}{n^{1 / q}}\right|=\left|\left\langle\left(b_{n} n^{-1 / q}\right)_{n=1}^{\infty},\left(a_{n}\right)_{n=1}^{\infty}\right\rangle\right| \leq\left\|\left(b_{n} n^{-1 / q}\right)_{n=1}^{\infty}\right\|_{\text {ces }_{p}^{*}} \cdot\|a\|_{\text {ces }_{p}} .
$$

Let $c=\left(c_{n}\right)_{n=1}^{\infty}$ with $c_{n}:=\frac{\left|a_{n}\right| \overline{b_{n}}}{\left|b_{n}\right|}$ if $b_{n} \neq 0$ and $c_{n}=0$ in other case. Then $\left|c_{n}\right| \leq\left|a_{n}\right|$ for all $n \geq 1$. Since $a \in c e s_{p}$, it follows that $c \in \operatorname{ces}_{p}$ and $\|c\|_{\text {ces }_{p}} \leq\|a\|_{c e s_{p}}$. Hence,

$$
\begin{aligned}
\sum_{n=1}^{\infty} \frac{\left|a_{n} b_{n}\right|}{n^{1 / q}}=\left|\sum_{n=1}^{\infty} \frac{c_{n} b_{n}}{n^{1 / q}}\right| & \leq\left\|\left(b_{n} n^{-1 / q}\right)_{n=1}^{\infty}\right\|_{{\text {ces } s_{p}^{*}}^{*}} \cdot\|c\|_{\text {ces }_{p}} \\
& \leq\left\|\left(b_{n} n^{-1 / q}\right)_{n=1}^{\infty}\right\|_{c e s_{p}^{*}} \cdot\|a\|_{\text {ces }_{p}}
\end{aligned}
$$

So $T_{b}(a) \in \ell_{q}^{1}$.

Example 5.3. For $\alpha>\frac{1}{q}$ set $b_{n}=(\log n)^{-\alpha}$, for $n \geq 2$. Then

$$
\sum_{n=2}^{\infty} \sup _{k \geq n}\left(\frac{\left|(\log k)^{-\alpha}\right|^{q}}{k}\right)=\sum_{n=2}^{\infty} \frac{1}{n(\log n)^{q \alpha}}<\infty
$$

Thus, for every $\sum_{n=1}^{\infty} a_{n} n^{-s} \in \mathcal{H}\left(\right.$ ces $\left._{p}\right)$ we have that

$$
\sum_{n=2}^{\infty} \frac{a_{n}}{n^{s} \log ^{\alpha} n}
$$

is a multiplier on $\mathcal{H}\left(c e s_{p}\right)$.

\section{Acknowledgment}

The authors thank the referee for his/her valuable suggestions.

\section{References}

[1] S.V. Astashkin, L. Maligranda, Interpolation of Cesàro sequence and function spaces, Studia Math. 215 (2013) 39-69.

[2] M. Bailleul, Espaces de Banach de séries de Dirichlet et leurs opérateurs de composition, PhD thesis, Université d'Artois, 2014.

[3] M. Bailleul, O.F. Brevig, Composition operators on Bohr-Bergman spaces of Dirichlet series, Ann. Acad. Sci. Fenn. Math. 41 (2016) 129-142.

[4] M. Bailleul, P. Lefèvre, Some Banach spaces of Dirichlet series, Studia Math. 226 (2015) 17-55.

[5] R. Balasubramanian, B. Calado, H. Queffélec, The Bohr inequality for ordinary Dirichlet series, Studia Math. 175 (2006) 285-304.

[6] F. Bayart, Hardy spaces of Dirichlet series and their composition operators, Monatsh. Math. 136 (2002) $203-236$.

[7] F. Bayart, C. Finet, D. Li, H. Queffélec, Composition operators on the Wiener-Dirichlet algebra, J. Operator Theory 60 (2008) 45-70.

[8] G. Bennett, Factorizing the classical inequalities, Mem. Amer. Math. Soc. 120 (576) (1996) 1-130.

[9] H. Bohr, Bidrag til de Dirichlet'ske Rækkers Theori (Contributions to the Theory of Dirichlet Series), PhD thesis, University of Copenhagen, 1910; Collected Mathematical Works, vol. III, Dansk Matematisk Forening, København, 1952 (in English), Document S-1.

[10] H. Bohr, Über die gleichmässige Konvergenz Dirichletscher Reihen, J. Reine Angew. Math. 143 (1913) $203-211$.

[11] R.M. Corless, G.H. Gonnet, D.E.G. Hare, D.J. Jeffrey, D.E. Knuth, On the Lambert W function, Adv. Comput. Math. 5 (1996) 329-359

[12] G.P. Curbera, W.J. Ricker, Solid extensions of the Cesàro operator on the Hardy space $H^{2}(\mathbb{D})$, J. Math. Anal. Appl. 407 (2013) 387-397. 
[13] G.P. Curbera, W.J. Ricker, Solid extensions of the Cesàro operator on $\ell^{p}$ and $c_{0}$, Integral Equations Operator Theory 80 (2014) 61-77.

[14] P.J. Davis, P. Rabinowitz, Methods of Numerical Integration, Academic Press, New York, 1984.

[15] G.H. Hardy, J.E. Littlewood, G. Pólya, Inequalities, Cambridge University Press, Cambridge, 1934.

[16] G.H. Hardy, M. Riesz, The General Theory of Dirichlet's Series, Cambridge University Press, Cambridge, 1915.

[17] H. Hedenmalm, P. Lindqvist, K. Seip, A Hilbert space of Dirichlet series and systems of dilated functions in $L^{2}(0,1)$, Duke Math. J. 86 (1997) 1-37.

[18] A.A. Jagers, A note on Cesàro sequence spaces, Nieuw Arch. Wiskd. (3) 22 (1974) 113-124.

[19] J.E. McCarthy, Hilbert spaces of Dirichlet series and their multipliers, Trans. Amer. Math. Soc. 356 (2004) 881-893.

[20] H. Queffélec, M. Queffélec, Diophantine Approximation and Dirichlet Series, Hindustan Book Agency, New Delhi, 2013.

[21] I. Schur, Über Potenzreihen, die im Innern des Einheitskreises beschränkt sind, J. Reine Angew. Math. 147 (1917) $205-232$.

[22] E.C. Titchmarsh, The Theory of Functions, Oxford University Press, Oxford, 1939. 